\title{
Aerial Co-Manipulation with Cables: The Role of Internal Force for Equilibria, Stability, and Passivity
}

\author{
Marco Tognon ${ }^{1}$, Chiara Gabellieri ${ }^{1,2, \dagger}$, Lucia Pallottino ${ }^{2}$, and Antonio Franchi ${ }^{1}$
}

\begin{abstract}
This paper considers the cooperative manipulation of a cable-suspended load with two generic aerial robots without the need of explicit communication. The role of the internal force for the asymptotic stability of the beam position-andattitude equilibria is analyzed in depth. Using a nonlinear Lyapunov-based approach, we prove that if a non-zero internal force is chosen, then the asymptotic stabilization of any desired beam attitude can be achieved with a decentralized and communication-less master-slave admittance controller. If, conversely, a zero internal force is chosen, as done in the majority of the state-of-the-art algorithms, the attitude of the beam is not controllable without communication. Furthermore, we formally proof the output-strictly passivity of the system with respect to an energy-like storage function and a certain input-output pair. This proves the stability and the robustness of the method during motion and in non-ideal conditions. The theoretical findings are validated through extensive simulations.
\end{abstract}

\section{INTRODUCTION}

Over the last decade UAVs (Unmanned Aerial Vehicles) have risen the interest of a larger and larger audience for their wide application domain. Recently, aerial physical interaction, using aerial manipulators [1], [2] or exploiting physical links as cables [3], has become a very popular topic. One interesting and applicative problem is the aerial manipulation of large objects, for which cooperative approaches are usually applied because they allow to overcome the limited payload of a single platform, thus lifting larger and heavier loads [4].

Many works targeted this problem proposing different methods and solutions. In [5], [6] cooperative aerial transportation of a rigid and an elastic object is considered, respectively. In [7] the use of multiple flying arms is exploited to address the problem. Aerial manipulation via cables is another interesting solution to the problem since it can reduce the couplings between the load and the robot attitude dynamics. Examples of cooperative aerial manipulation using cables are studied in [8]-[10]. All these examples rely on a centralized control. Instead, a decentralized algorithm, as in [11], is more robust and scalable with respect to (w.r.t.) the number of robots.

\footnotetext{
The first two authors have equally collaborated to the manuscript, and can both be considered as first author.

${ }^{1}$ LAAS-CNRS, Université de Toulouse, CNRS, Toulouse, France, antonio.franchi@laas.fr, marco.tognon@laas.fr, cgabellieri@laas.fr

${ }^{2}$ Centro di ricerca E. Piaggio, Università di Pisa, Largo Lucio Lazzarino 1, 56122,Pisa, Italy lucia.pallottino@unipi.it

This work has been funded by the European Union's Horizon 2020 research and innovation program under grant agreement No 644271 AEROARMS
}

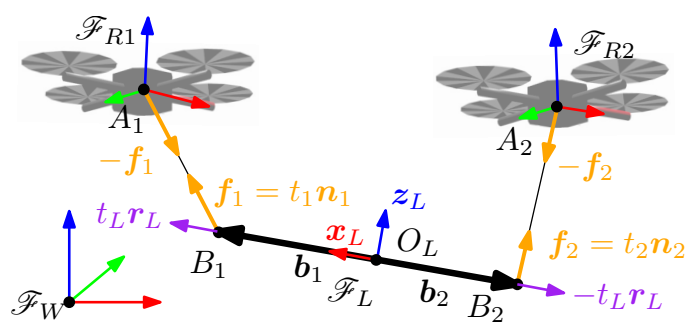

Fig. 1: Representation of the system and its major variables. The two aerial vehicles do not need to be necessarily quadrotors since the analysis and control design is valid for general aerial vehicles.

However, the major bottleneck in decentralized algorithms is the explicit communication. Communication delays and packet losses can affect the performance and even the stability of the systems. Limiting the need for explicit communication allows to reduce the complexity as well. In [12] the authors proposed one of the first decentralized leaderfollower algorithm without explicit communication, for objects transportation performed by mobile ground robots. Aerial cooperative transportation by two robots without explicit communication has been addressed also in [13] for a cable-suspended beam-like load, and a leader-follower paradigm has been proposed. Here the leader follows an external position reference, while the horizontal position of the follower is controlled with an admittance filter, trying to keep the cable always vertical (zero internal force). A similar approach has been proposed in [14] but relying on a visual feedback. However, those methods do not deal with the load pose control and do not provide a formal stability proof.

For the same system composed by two aerial robots carrying a cable suspended beam-like load (see Fig. 1 for a schematic representation), we propose a decentralized algorithm relying only on implicit communication. Our algorithm uses a master-slave architecture with an admittance filter on both robots (not only on the slave as in the related state of the art), to make the overall system compliant/robust to external disturbances.

One of our main contributions is the constructive and intuitive method to choose the controller input to stabilize the load at a desired pose. The control of both position and orientation turns the simpler transportation task found in the state of the art in a full-manipulation one.

We show that those inputs are parametrized by the internal force of the load that plays a crucial role in the equilibria stability. Differently from the state of the art algorithms, which are not formally guaranteed to converge, we also provide a formal proof of the stability through Lyapunov's 
direct method. Furthermore, we prove that the controlled system is output-strictly passive w.r.t. a relevant input-output pair. This provides a bound for the energy variations during the manipulation and an index of robustness of the method.

In Sec. II we derive the model. In Sec. III we present the control strategy and the equilibria of the system. Their stability is discussed in Sec. IV. In Sec. V we prove the passivity and stability of transportation. Simulation results and conclusive discussions are presented in Sec. VI and VII, respectively.

\section{System Modeling}

The considered system and its major variables are shown in Fig. 1. The beam-like load is modeled as a rigid body with mass $m_{L} \in \mathbb{R}_{>0}$ and a positive definite inertia matrix $\boldsymbol{J}_{L} \in$ $\mathbb{R}^{3 \times 3}$. We define the frame $\mathscr{F}_{L}=\left\{\boldsymbol{O}_{L}, \boldsymbol{x}_{L}, \boldsymbol{y}_{L}, \boldsymbol{z}_{L}\right\}$ rigidly attached to it, where $O_{L}$ is the the load center of mass (CoM). Then, we define an inertial frame $\mathscr{F}_{W}=\left\{\boldsymbol{O}_{W}, \boldsymbol{x}_{W}, \boldsymbol{y}_{W}, \boldsymbol{z}_{W}\right\}$ with $z_{W}$ oriented in the opposite direction to the gravity vector. The configuration of the load is then described by the position of $O_{L}$ and orientation of $\mathscr{F}_{L}$ with respect to $\mathscr{F}_{W}$, i.e., by the vector $\boldsymbol{p}_{L} \in \mathbb{R}^{3}$ and the rotation matrix $\boldsymbol{R}_{L} \in$ $S O(3)$, respectively. Its dynamics is given by the NewtonEuler equations

$$
\begin{aligned}
m_{L} \ddot{\boldsymbol{p}}_{L} & =-m_{L} g \boldsymbol{e}_{3}+\boldsymbol{f}_{e} \\
\dot{\boldsymbol{R}}_{L} & =\boldsymbol{S}\left(\boldsymbol{\omega}_{L}\right) \boldsymbol{R}_{L} \\
\boldsymbol{J}_{L} \dot{\boldsymbol{\omega}}_{L} & =-\boldsymbol{S}\left(\boldsymbol{\omega}_{L}\right) \boldsymbol{J}_{L} \boldsymbol{\omega}_{L}+\boldsymbol{\tau}_{e}-\boldsymbol{\omega}_{L}^{\top} \boldsymbol{B}_{L} \boldsymbol{\omega}_{L}
\end{aligned}
$$

where, $\omega_{L} \in \mathbb{R}^{3}$ is the angular velocity of $\mathscr{F}_{L}$ w.r.t. $\mathscr{F}_{W}$ expressed in $\mathscr{F}_{L}, \boldsymbol{S}(\star)$ is the operator such that $\boldsymbol{S}(\boldsymbol{x}) \boldsymbol{y}=$ $\boldsymbol{x} \times \boldsymbol{y}, g$ is the gravitational constant, $\boldsymbol{e}_{i}$ is the canonical unit vector with a 1 in the $i$-th entry, $f_{e}$ and $\tau_{e} \in \mathbb{R}^{3}$ are the sum of external forces and moments acting on the load, respectively. The positive definite matrix $\boldsymbol{B}_{L} \in \mathbb{R}^{3 \times 3}$ is a damping factor modeling the energy dissipation phenomena.

The load is transported by two aerial robots by means of two cables, one for each robot. We denote with $A_{i}$ the attachment point of the $i$-th cable to the $i$-th robot, with $i=1,2$, and we define the frame $\mathscr{F}_{R i}=\left\{A_{i}, \boldsymbol{x}_{R i}, \boldsymbol{y}_{R i}, \boldsymbol{z}_{R i}\right\}$ rigidly attached to the robot and centered in the attachment point. The $i$-th robot configuration is described by the position of $A_{i}$ and orientation of $\mathscr{F}_{R i}$ w.r.t. $\mathscr{F}_{W}$, denoted by the vector $\boldsymbol{p}_{R i} \in \mathbb{R}^{3}$, and the rotation matrix $\boldsymbol{R}_{R i} \in S O(3)$, respectively. We assume that a position controller is applied to the aerial robot, able to track any $C^{2}$ trajectory with negligible error in the domain of interest, independently from external disturbances. Indeed, with the recent robust controllers (as the one in [15] for both unidirectional- and multidirectional-thrust vehicles) and disturbance observers for aerial vehicles, one can obtain very precise motions, even in the presence of external disturbances. However, the proposed control method results particularly robust to non-ideality, thanks to its passivity nature (see Sec. V). As a consequence, in real applications, a precise tracking is actually not needed for the stability, but only to achieve perfect performance.
The closed loop translational dynamics of the robot subject to the position controller is then assumed as the one of a double integrator: $\ddot{\boldsymbol{p}}_{R i}=\boldsymbol{u}_{R i}$, where $\boldsymbol{u}_{R i}$ is a virtual input to be designed. If we consider a multidirectional-thrust platform capable of controlling both position and orientation independently [16], the double integrator is an exact model of the closed loop system apart from modeling errors. In the case of underactuated unidirectional-thrust vehicle, the double integrator is instead a very good approximation. Indeed the rotational dynamics is totally decoupled from the translational one and it is much faster than the latter, allowing to apply the time-scale separation principle. At this stage it might seem that the platform is 'infinitely stiff' w.r.t. the force produced by the cable. However, we shall re-introduce a compliant behavior by suitably designing the input $\boldsymbol{u}_{R i}$.

The other end of the $i$-th cable is attached to the load at the anchoring point $B_{i}$ described by the vector ${ }^{L} \boldsymbol{b}_{i} \in \mathbb{R}^{3}$ denoting its position with respect to $\mathscr{F}_{L}$. The position of $B_{i}$ w.r.t. $\mathscr{F}_{W}$ is then given by $\boldsymbol{b}_{i}=\boldsymbol{p}_{L}+\boldsymbol{R}_{L}{ }^{L} \boldsymbol{b}_{i}$. To simplify the discussion we assume, without loss of generality, that ${ }^{L} \boldsymbol{b}_{1}=\left[\left\|{ }^{L} \boldsymbol{b}_{1}\right\| \quad 00\right]^{\top}$.

Assumption 1. The two anchoring points are placed such that the load CoM coincides with their middle point, i.e., ${ }^{L} b_{1}=$ $-{ }^{L} \boldsymbol{b}_{2}$. This assumption is rather easy to meet in practice.

We model the $i$-th cable as a unilateral spring along its principal direction, characterized by a constant elastic coefficient $k_{i} \in \mathbb{R}_{>0}$, a constant nominal length denoted by $l_{0 i}$ and a negligible mass and inertia w.r.t. the ones of the robots and of the load. The attitude of the cable is described by the normalized vector, $\boldsymbol{n}_{i}=\boldsymbol{l}_{i} /\left\|\boldsymbol{l}_{i}\right\|$, where $\boldsymbol{l}_{i}=\boldsymbol{p}_{R i}-\boldsymbol{b}_{i}$. Given a certain elongation $\left\|l_{i}\right\|$ of the cable, the latter produces a force acting on the load at $B_{i}$ equal to:

$$
\boldsymbol{f}_{i}=t_{i} \boldsymbol{n}_{i}, \quad t_{i}=\left\{\begin{array}{ll}
k_{i}\left(\left\|\boldsymbol{l}_{i}\right\|-l_{0 i}\right) & \text { if }\left\|\boldsymbol{l}_{i}\right\|-l_{0 i}>0 \\
0 & \text { otherwise }
\end{array} .\right.
$$

$t_{i} \in \mathbb{R}_{>0}$ denotes the tension along the cable and it is given by the simplified Hooke's law. As usually done in the related literature, we assume that the controller and the gravity force always maintain the cables taut, at least in the domain of interest. The force produced at the other hand of the cable, namely on the $i$-th robot at $A_{i}$, is equal to $-f_{i}$.

Considering the forces that robots and load exchange by means of the cables, the dynamics of the full system is:

$$
\begin{aligned}
& \dot{\boldsymbol{v}}_{R}=\boldsymbol{u}_{R} \\
& \dot{\boldsymbol{v}}_{L}=\boldsymbol{M}_{L}^{-1}\left(-\boldsymbol{c}_{L}\left(\boldsymbol{v}_{L}\right)-\boldsymbol{g}_{L}+\boldsymbol{G}\left(\boldsymbol{q}_{L}\right) \boldsymbol{f}\right),
\end{aligned}
$$

where $\boldsymbol{q}_{R}=\left[\begin{array}{ll}\boldsymbol{p}_{R 1}^{\top} & \boldsymbol{p}_{R 2}^{\top}\end{array}\right]^{\top}, \boldsymbol{q}_{L}=\left(\boldsymbol{p}_{L}, \boldsymbol{R}_{L}\right), \boldsymbol{v}_{R}=\left[\begin{array}{ll}\dot{\boldsymbol{p}}_{R 1}^{\top} & \dot{\boldsymbol{p}}_{R 2}^{\top}\end{array}\right]^{\top}$, $\boldsymbol{v}_{L}=\left[\begin{array}{ll}\dot{\boldsymbol{p}}_{L}^{\top} & \boldsymbol{\omega}_{L}^{\top}\end{array}\right]^{\top}, \boldsymbol{u}_{R}=\left[\begin{array}{ll}\boldsymbol{u}_{R 1}^{\top} & \boldsymbol{u}_{R 2}^{\top}\end{array}\right]^{\top}, \boldsymbol{f}=\left[\begin{array}{ll}\boldsymbol{f}_{1}^{\top} & \boldsymbol{f}_{2}^{\top}\end{array}\right]^{\top}$ where $\boldsymbol{f}_{i}$ is given in (1), and is a function of the state, $\boldsymbol{M}_{L}=$ $\operatorname{diag}\left(m_{L} \boldsymbol{I}_{3}, \boldsymbol{J}_{L}\right)$ and $\boldsymbol{I}_{3} \in \mathbb{R}^{3 \times 3}$ the identity matrix, $\boldsymbol{g}_{L}=$

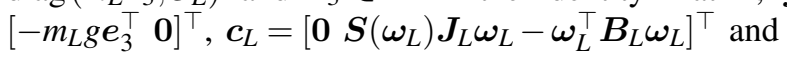

$$
\boldsymbol{G}=\left[\begin{array}{cc}
\boldsymbol{I}_{3} & \boldsymbol{I}_{3} \\
\boldsymbol{S}\left({ }^{L} \boldsymbol{b}_{1}\right) \boldsymbol{R}_{L}^{\top} & \boldsymbol{S}\left({ }^{L} \boldsymbol{b}_{2}\right) \boldsymbol{R}_{L}^{\top}
\end{array}\right]
$$

We remark that the two dynamics in (2) are coupled together by the cable forces in (1). 


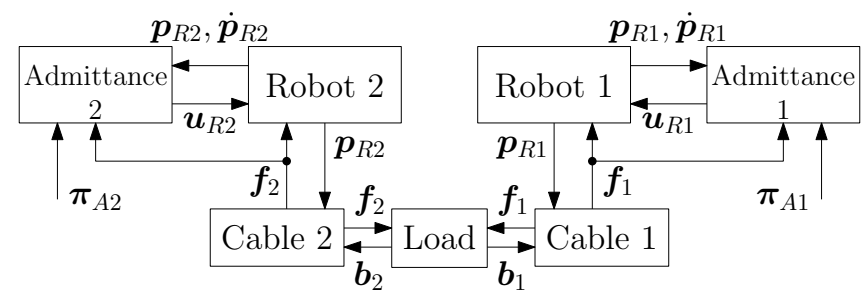

Fig. 2: Schematic representation of the overall system including both physical and control blocks.

\section{Control problem}

In this work we aim to: i) stabilize the load at a desired configuration, $\overline{\boldsymbol{q}}_{L}=\left(\overline{\boldsymbol{p}}_{L}, \overline{\boldsymbol{R}}_{L}\right)$; ii) preserve the stability of the load during its transportation.

Assuming a perfect knowledge of the system dynamic model, and a perfect state estimation, one could use a centralized control approach, as in [8], [9]. We are instead interested in solving the mentioned objectives using a decentralized approach without explicit communication between the robots.

\section{CONTROL DESIGN AND EQUILIBRIA}

To achieve the previous control objectives we propose the use of an admittance filter for both robots, i.e., setting:

$$
\boldsymbol{u}_{R i}=\boldsymbol{M}_{A i}^{-1}\left(-\boldsymbol{B}_{A i} \dot{\boldsymbol{p}}_{R i}-\boldsymbol{K}_{A i} \boldsymbol{p}_{R i}-\boldsymbol{f}_{i}+\boldsymbol{\pi}_{A i}\right),
$$

where the tree positive definite symmetric matrices $\boldsymbol{M}_{A i}, \boldsymbol{B}_{A i}, \boldsymbol{K}_{A i} \in \mathbb{R}^{3 \times 3}$ are the virtual inertia of the robot, the virtual damping, and the stiffness of a virtual spring attached to the robot, and $\pi_{A i} \in \mathbb{R}^{3}$ is an additional input (see Fig. 2 for a schematic representation). Notice that (3) does not require explicit communication. Indeed it requires only local information, i.e., the state of the robot $\left(\boldsymbol{p}_{R i}, \dot{\boldsymbol{p}}_{R i}\right)$, and the force applied by the cable $f_{i}$. The first can be retrieved with standard on-board sensors, while the second can be directly measured by an on-board force sensor or estimated by a sufficiently precise model-based observer as done in [13], [16].

Combining equations (2) and (3) we can write the closed loop system dynamics as $\dot{\boldsymbol{v}}=m\left(\boldsymbol{q}, \boldsymbol{v}, \boldsymbol{\pi}_{A}\right)$ where

$$
m\left(\boldsymbol{q}, \boldsymbol{v}, \boldsymbol{\pi}_{A}\right)=\left[\begin{array}{c}
\boldsymbol{M}_{A}^{-1}\left(-\boldsymbol{B}_{A} \dot{\boldsymbol{p}}_{R}-\boldsymbol{K}_{A} \boldsymbol{p}_{R}-\boldsymbol{f}+\boldsymbol{\pi}_{A}\right) \\
\boldsymbol{M}_{L}^{-1}\left(-\boldsymbol{c}_{L}\left(\boldsymbol{v}_{L}\right)-\boldsymbol{g}_{L}+\boldsymbol{G} \boldsymbol{f}\right)
\end{array}\right],
$$

with $\boldsymbol{q}=\left(\boldsymbol{q}_{R}, \boldsymbol{q}_{L}\right), \boldsymbol{v}=\left[\boldsymbol{v}_{R}^{\top} \boldsymbol{v}_{L}^{\top}\right]^{\top}$ and $\boldsymbol{\pi}_{A}=\left[\boldsymbol{\pi}_{A 1}^{\top} \boldsymbol{\pi}_{A 2}^{\top}\right]^{\top}$. Furthermore $\boldsymbol{M}_{A}=\operatorname{diag}\left(\boldsymbol{M}_{A 1}, \boldsymbol{M}_{A 2}\right), \boldsymbol{B}_{A}=\operatorname{diag}\left(\boldsymbol{B}_{A 1}, \boldsymbol{B}_{A 2}\right)$ and $\boldsymbol{K}_{A}=\operatorname{diag}\left(\boldsymbol{K}_{A 1}, \boldsymbol{K}_{A 2}\right)$. In order to coordinate the motions of the robots in a decentralized way we propose a master-slave approach. Only one robot, namely the designated master, will have an active control of the system. Choosing robot 1 as master and robot 2 as slave we set $\boldsymbol{K}_{A 1} \neq \mathbf{0}, \boldsymbol{K}_{A 2}=\mathbf{0}$.

We say that $\boldsymbol{q}$ is an equilibrium configuration if $\exists \boldsymbol{\pi}_{A}$ s.t. $\mathbf{0}=m\left(\boldsymbol{q}, \mathbf{0}, \boldsymbol{\pi}_{A}\right)$, i.e, if the corresponding zero-velocity state $(\boldsymbol{q}, \mathbf{0})$ is a forced equilibrium for the system (4) for a certain forcing input $\boldsymbol{\pi}_{A}$. We say that an equilibrium configuration $\boldsymbol{q}$ is stable, unstable, or asymptotically stable if $(\boldsymbol{q}, \mathbf{0})$ is stable, unstable, or asymptotically stable, respectively.

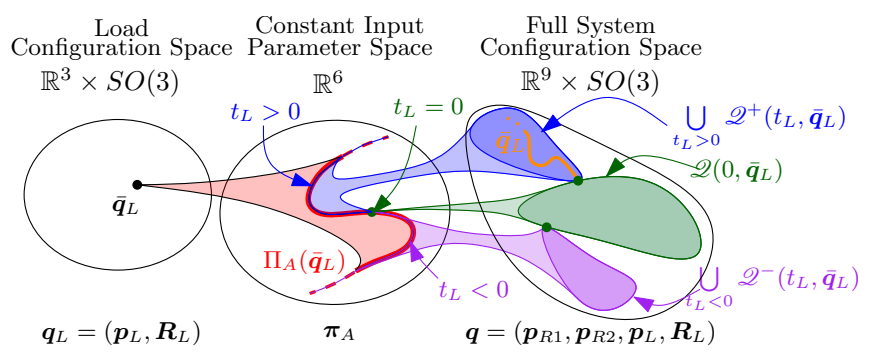

Fig. 3: Relation between the equilibria and forcing control input. In particular, starting from the left: to a desired load configuration of equilibrium it corresponds a forcing input in the subset $\Pi_{A}\left(\overline{\boldsymbol{q}}_{L}\right)$ of dimension one (inverse problem). Then, moving to the right: to a forcing input in $\Pi_{A}\left(\overline{\boldsymbol{q}}_{L}\right)$ it corresponds an equilibrium in the subsets $\mathscr{Q}^{+}\left(t_{L}, \overline{\boldsymbol{q}}_{L}\right), \mathscr{Q}^{-}\left(t_{L}, \overline{\boldsymbol{q}}_{L}\right)$ or $\mathscr{Q}\left(0, \overline{\boldsymbol{q}}_{L}\right)$ according to the value of $t_{L}$ (direct problem). The orange line inside $\mathscr{Q}^{+}\left(t_{L}, \overline{\boldsymbol{q}}_{L}\right)$ corresponds to the equilibria $\boldsymbol{q} \in \mathscr{Q}^{+}\left(t_{L}, \overline{\boldsymbol{q}}_{L}\right)$ such that $\boldsymbol{q}_{L}=\overline{\boldsymbol{q}}_{L}$.

In the following we shall prove that for any desired load configuration $\overline{\boldsymbol{q}}_{L}$ there exists a set $\Pi_{A}\left(\overline{\boldsymbol{q}}_{L}\right) \subset \mathbb{R}^{6}$ such that for any $\boldsymbol{\pi}_{A} \in \Pi_{A}\left(\overline{\boldsymbol{q}}_{L}\right)$ one can compute a $\overline{\boldsymbol{q}}_{R}$, depending on $\overline{\boldsymbol{q}}_{L}$ and $\boldsymbol{\pi}_{A}$, that makes $\overline{\boldsymbol{q}}=\left(\overline{\boldsymbol{q}}_{L}, \overline{\boldsymbol{q}}_{R}\right)$ an asymptotically stable equilibrium with $\pi_{A}$ as forcing input. As we shall see, a key role in all the following analyses is played by the load internal force, defined as

$$
t_{L}:=\frac{1}{2} \boldsymbol{f}^{\top}\left[\boldsymbol{I}_{3}-\boldsymbol{I}_{3}\right]^{\top} \boldsymbol{R}_{L} \boldsymbol{e}_{1}=: \frac{1}{2} \boldsymbol{f}^{\top} \boldsymbol{r}_{L}
$$

where $\boldsymbol{r}_{L}=\left[\begin{array}{ll}\boldsymbol{I}_{3} & -\boldsymbol{I}_{3}\end{array}\right]^{\top} \overline{\boldsymbol{R}}_{L} \boldsymbol{e}_{1}$. We have that if $t_{L}>0$ the internal force is a tension (the work of the internal force is positive if the distance between the anchoring points increases) while if $t_{L}<0$ the internal force is a compression (viceversa, the work is positive if the distance decreases).

\section{A. Equilibrium Configurations of the Closed Loop System}

We firstly carefully analyze the relation between equilibrium configurations, from now on simply called equilibria, and the forcing input $\boldsymbol{\pi}_{A}$. In particular, we shall study: i) equilibria inverse problem: which is the set of inputs (and corresponding robot positions) that equilibrates a desired $\overline{\boldsymbol{q}}_{L}$ (Theorem 1); ii) equilibria direct problem: which is the set of equilibria if $\pi_{A}$, chosen in the aforementioned set, is applied to the system (Theorem 2). A schematic representation of the results described in the theorems is given in Fig. 3.

Theorem 1 (equilibria inverse problem). Consider the closed loop system (4) and assume that the load is at a given desired configuration $\boldsymbol{q}_{L}=\overline{\boldsymbol{q}}_{L}=\left(\overline{\boldsymbol{p}}_{L}, \overline{\boldsymbol{R}}_{L}\right)$. For each internal force $t_{L} \in \mathbb{R}$, there exists an unique constant value for the forcing input $\boldsymbol{\pi}_{A}=\overline{\boldsymbol{\pi}}_{A}$ (and an unique position of the robots $\boldsymbol{q}_{R}=\overline{\boldsymbol{q}}_{R}$ ) such that $\overline{\boldsymbol{q}}=\left(\overline{\boldsymbol{q}}_{L}, \overline{\boldsymbol{q}}_{R}\right)$ is an equilibrium of the system.

In particular $\overline{\boldsymbol{\pi}}_{A}$ and $\overline{\boldsymbol{q}}_{R}=\left[\overline{\boldsymbol{p}}_{R 1}^{\top} \overline{\boldsymbol{p}}_{R 2}^{\top}\right]^{\top}$ are given by

$$
\begin{aligned}
& \overline{\boldsymbol{\pi}}_{A}\left(\overline{\boldsymbol{q}}_{L}, t_{L}\right)=\boldsymbol{K}_{A} \overline{\boldsymbol{q}}_{R}+\overline{\boldsymbol{f}}\left(\overline{\boldsymbol{q}}_{L}, t_{L}\right) \\
& \overline{\boldsymbol{p}}_{R i}\left(\overline{\boldsymbol{q}}_{L}, t_{L}\right)=\overline{\boldsymbol{p}}_{L}+\overline{\boldsymbol{R}}_{L}{ }^{L} \boldsymbol{b}_{i}+\left(\frac{\left\|\overline{\boldsymbol{f}}_{i}\right\|}{k_{i}}+l_{0 i}\right) \frac{\overline{\boldsymbol{f}}_{i}}{\left\|\overline{\boldsymbol{f}}_{i}\right\|},
\end{aligned}
$$

for $i=1,2$, where

$$
\overline{\boldsymbol{f}}\left(\overline{\boldsymbol{q}}_{L}, t_{L}\right)=\left[\begin{array}{l}
\overline{\boldsymbol{f}}_{1} \\
\overline{\boldsymbol{f}}_{2}
\end{array}\right]=\frac{m_{L} g}{2}\left[\begin{array}{l}
\boldsymbol{I}_{3} \\
\boldsymbol{I}_{3}
\end{array}\right] \boldsymbol{e}_{3}+t_{L}\left[\begin{array}{c}
\boldsymbol{I}_{3} \\
-\boldsymbol{I}_{3}
\end{array}\right] \overline{\boldsymbol{R}}_{L} e_{1}
$$


Proof. The desired load configuration $\bar{q}_{L}$ can be equilibrated if there exists at least a $\overline{\boldsymbol{q}}_{R}$ and a $\boldsymbol{\pi}_{A}$ such that:

$$
m\left(\overline{\boldsymbol{q}}, \mathbf{0}, \boldsymbol{\pi}_{A}\right)=\mathbf{0} .
$$

Consider the last six rows of (9). We must find the $f$ solving

$$
\boldsymbol{G} \boldsymbol{f}=\boldsymbol{g}_{L} \text {. }
$$

$\boldsymbol{G}$ is not invertible since $\operatorname{rank}(\boldsymbol{G})=5$, thus we have to verify that a solution for (10) exists. Expanding (10) we obtain

$$
\begin{aligned}
\boldsymbol{f}_{1}+\boldsymbol{f}_{2} & =-m_{L} \boldsymbol{g} \boldsymbol{e}_{3} \\
\boldsymbol{S}\left({ }^{L} \boldsymbol{b}_{1}\right) \overline{\boldsymbol{R}}_{L}^{\top} \boldsymbol{f}_{1}+\boldsymbol{S}\left({ }^{L} \boldsymbol{b}_{2}\right) \overline{\boldsymbol{R}}_{L}^{\top} \boldsymbol{f}_{2} & =\mathbf{0 .} .
\end{aligned}
$$

Then, substituting in (12) the $f_{1}$ obtained from (11) we have $2 \boldsymbol{S}\left({ }^{L} \boldsymbol{b}_{1}\right) \overline{\boldsymbol{R}}_{L}^{\top} \boldsymbol{f}_{2}=-\boldsymbol{S}\left({ }^{L} \boldsymbol{b}_{1}\right) \overline{\boldsymbol{R}}_{L}^{\top} m_{L} g \boldsymbol{e}_{3}$, for which $\boldsymbol{f}_{2}=$ $m_{L} g e_{3} / 2$ is always a solution. Therefore, all the solutions of (10) can be written as

$$
\overline{\boldsymbol{f}}=\boldsymbol{G}^{\dagger} \boldsymbol{g}_{L}+\boldsymbol{r}_{L} t_{L},
$$

where $\boldsymbol{G}^{\dagger}=1 / 2\left[\begin{array}{ll}\boldsymbol{I}_{3} & \boldsymbol{I}_{3}\end{array}\right]^{\top}$ is the pseudo inverse of $\boldsymbol{G}, \boldsymbol{r}_{L} \in \mathbb{R}^{6}$ is a vector in $\operatorname{Null}(\boldsymbol{G})$, and $t_{L} \in \mathbb{R}$ is an arbitrary number.

We computed $\boldsymbol{r}_{L}=\left[\boldsymbol{f}_{1}^{\top} \boldsymbol{f}_{2}^{\top}\right]^{\top}$ from (11) and (12) imposing the right hand side equal to zero. From (11) $f_{2}=-f_{1}$, and replacing it into (12) we obtain $\boldsymbol{S}\left(2^{L} \boldsymbol{b}_{1}\right) \overline{\boldsymbol{R}}_{L}^{\top} \boldsymbol{f}_{1}=\mathbf{0}$ which is verified if $f_{1}=t_{L} \overline{\boldsymbol{R}}_{L} \boldsymbol{e}_{1}$ with $t_{L} \in \mathbb{R}$. Finally we obtain $\boldsymbol{r}_{L}=\left[\begin{array}{ll}\boldsymbol{I}_{3} & -\boldsymbol{I}_{3}\end{array}\right]^{\top} \overline{\boldsymbol{R}}_{L} \boldsymbol{e}_{1}$, as in the definition (5).

Equation (13) can be then rewritten as (8). The expression of $\bar{p}_{R i}$ in (7) is computed using (1) and the kinematics of the system. Notice that (7) is singular when $\bar{f}_{i}=\mathbf{0}$ for some $i$. However this can always be avoided properly choosing $t_{L}$.

Lastly, from the first six rows of (9) we have that $\overline{\boldsymbol{q}}_{L}$ is equilibrated if $\boldsymbol{\pi}_{A}=\overline{\boldsymbol{\pi}}_{A}$, where $\overline{\boldsymbol{\pi}}_{A}$ is defined as in (6).

Remark 1. Based on Theorem 1 we can define a set $\Pi_{A}\left(\overline{\boldsymbol{q}}_{L}\right)=\left\{\boldsymbol{\pi}_{A} \in \mathbb{R}^{6}: \boldsymbol{\pi}_{A}=\overline{\boldsymbol{\pi}}_{A}\left(\overline{\boldsymbol{q}}_{L}, t_{L}\right)\right.$ for $\left.t_{L} \in \mathbb{R}\right\}$ which has dimension 1 , since it is parametrized by the scalar $t_{L} \in \mathbb{R}$.

Remark 2. Given a desired load configuration $\overline{\boldsymbol{q}}_{L}$ to equilibrate, Theorem 1 and its constructive proofs, give an intuitive method for choosing the forcing input $\boldsymbol{\pi}_{A}$. In particular one has to choose only the value of the internal force $t_{L}$.

Once $t_{L}$ is chosen and the input $\boldsymbol{\pi}_{A}=\overline{\boldsymbol{\pi}}_{A}\left(t_{L}, \overline{\boldsymbol{q}}_{L}\right)$ is applied to the system, it is not in general granted that $\left(\overline{\boldsymbol{q}}_{L}, \overline{\boldsymbol{q}}_{R}\right)$ is the only equilibrium of (4), i.e., the equilibria direct problem may have multiple solutions.

Theorem 2 (equilibria direct problem). Given $t_{L} \in \mathbb{R}$ and the corresponding $\overline{\boldsymbol{\pi}}_{A} \in \Pi_{A}\left(\overline{\boldsymbol{q}}_{L}\right)$ computed as in (6), the equilibria of the system (4), when the input $\boldsymbol{\pi}_{A}=\overline{\boldsymbol{\pi}}_{A}\left(t_{L}, \overline{\boldsymbol{q}}_{L}\right)$ is applied, are all and only the ones described by the following conditions

$$
\begin{array}{r}
t_{L} \boldsymbol{R}_{L} \boldsymbol{e}_{1} \times \overline{\boldsymbol{R}}_{L} \boldsymbol{e}_{1}=\mathbf{0} \\
\boldsymbol{p}_{R 1}=\overline{\boldsymbol{p}}_{R 1} \\
\boldsymbol{p}_{L}=\boldsymbol{p}_{R 1}-\boldsymbol{R}_{L}{ }^{L} \boldsymbol{b}_{1}-\left(\frac{\left\|\overline{\boldsymbol{f}}_{1}\right\|}{k_{1}}+l_{01}\right) \frac{\overline{\boldsymbol{f}}_{1}}{\left\|\overline{\boldsymbol{f}}_{1}\right\|}= \\
=\overline{\boldsymbol{p}}_{L}+\left(\overline{\boldsymbol{R}}_{L}-\boldsymbol{R}_{L}\right)^{L} \boldsymbol{b}_{1} \\
\boldsymbol{p}_{R 2}=\boldsymbol{p}_{L}+\boldsymbol{R}_{L}{ }^{L} \boldsymbol{b}_{2}+\left(\frac{\left\|\overline{\boldsymbol{f}}_{2}\right\|}{k_{2}}+l_{02}\right) \frac{\overline{\boldsymbol{f}}_{2}}{\left\|\overline{\boldsymbol{f}}_{2}\right\|}
\end{array}
$$

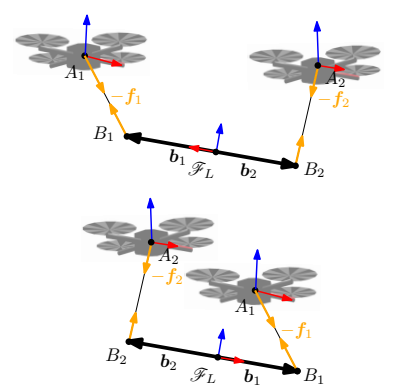

(a) Two equilibria for $t_{L} \neq 0$. On the top and on the bottom one equilibrium configuration in $\mathscr{Q}^{+}\left(t_{L}, \overline{\boldsymbol{q}}_{L}\right)$ and $\mathscr{Q}^{-}\left(t_{L}, \overline{\boldsymbol{q}}_{L}\right)$, respectively.

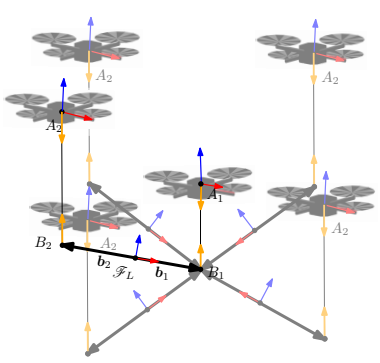

(b) Five of the possible infinite equilibria in $\mathscr{Q}\left(0, \overline{\boldsymbol{q}}_{L}\right)$. In vivid color the configuration $\overline{\boldsymbol{q}}$. The final load pose depends on the initial conditions.
Fig. 4: 2D representation of the equilibria varying $t_{L}$.

$\mathscr{Q}\left(t_{L}, \overline{\boldsymbol{q}}_{L}\right)$ denotes the set of configurations respecting (14).

Proof. Given $t_{L} \in \mathbb{R}$, and $\overline{\boldsymbol{\pi}}_{A} \in \Pi_{A}\left(\overline{\boldsymbol{q}}_{L}\right)$, a configuration $\boldsymbol{q}$ is an equilibrium if $m\left(\boldsymbol{q}, \mathbf{0}, \overline{\boldsymbol{\pi}}_{A}\right)=\mathbf{0}$. The first six rows are $\boldsymbol{K}_{A} \boldsymbol{q}_{R}+\boldsymbol{f}-\overline{\boldsymbol{\pi}}_{A}=\mathbf{0}$. Then, from (6) we have that

$$
\boldsymbol{f}=\boldsymbol{K}_{A}\left(\overline{\boldsymbol{q}}_{R}-\boldsymbol{q}_{R}\right)+\overline{\boldsymbol{f}} .
$$

Multiplying both sides of (15) by $G$ and using (10) we obtain $\boldsymbol{G} \boldsymbol{K}_{A}\left(\overline{\boldsymbol{q}}_{R}-\boldsymbol{q}_{R}\right)+\boldsymbol{G} \overline{\boldsymbol{f}}=\boldsymbol{g}_{L}$. Then, using $\boldsymbol{K}_{A 2}=\mathbf{0}$, and the expression of $\bar{f}$ in (8), we get

$$
\left[\begin{array}{c}
\boldsymbol{K}_{A 1} \boldsymbol{e}_{R 1} \\
\boldsymbol{S}\left({ }^{L} \boldsymbol{b}_{1}\right) \boldsymbol{R}_{L} \boldsymbol{K}_{A 1} \boldsymbol{e}_{R 1}
\end{array}\right]+\left[\begin{array}{c}
m_{L} g \boldsymbol{e}_{3} \\
2 \boldsymbol{S}\left({ }^{L} \boldsymbol{b}_{2}\right) \boldsymbol{R}_{L}^{\top} \overline{\boldsymbol{R}}_{L} \boldsymbol{e}_{1} t_{L}
\end{array}\right]=\left[\begin{array}{c}
m_{L} g \boldsymbol{e}_{3} \\
\mathbf{0}
\end{array}\right],
$$

where $\boldsymbol{e}_{R i}=\left(\overline{\boldsymbol{p}}_{R i}-\boldsymbol{p}_{R i}\right)$. The top row of (16) implies that $\boldsymbol{e}_{R 1}=\mathbf{0}$, hence $\boldsymbol{p}_{R 1}=\overline{\boldsymbol{p}}_{R 1}$. Replacing $\boldsymbol{e}_{R 1}=\mathbf{0}$ in the bottom part of (16) we obtain

$$
\begin{aligned}
\boldsymbol{S}\left({ }^{L} \boldsymbol{b}_{2}\right) \boldsymbol{R}_{L}^{\top} \overline{\boldsymbol{R}}_{L} \boldsymbol{e}_{1} t_{L}=\mathbf{0} & \Leftrightarrow{ }^{L} \boldsymbol{b}_{2} \times \boldsymbol{R}_{L}^{\top} \overline{\boldsymbol{R}}_{L} \boldsymbol{e}_{1} t_{L}=\mathbf{0} \\
& \Leftrightarrow \boldsymbol{R}_{L} \boldsymbol{e}_{1} \times \overline{\boldsymbol{R}}_{L} \boldsymbol{e}_{1} t_{L}=\mathbf{0} .
\end{aligned}
$$

We can retrieve $\boldsymbol{p}_{L}$ and $\boldsymbol{p}_{R 2}$, using (1) and the kinematics.

Remark 3. If $t_{L}=0$ the conditions in (17) hold for all the possible load attitudes $\boldsymbol{R}_{L} \in S O(3)$. This means that $\mathscr{Q}\left(0, \bar{q}_{L}\right)$ contains all the $\boldsymbol{R}_{L} \in S O(3)$ and the $\boldsymbol{q}_{R}, p_{L}$ computed from $\boldsymbol{R}_{L}$ using (14). Figure $4 \mathrm{~b}$ illustrates some of these equilibria.

For $t_{L} \neq 0$, it is required that $\boldsymbol{R}_{L} \boldsymbol{e}_{1}$ is parallel to $\overline{\boldsymbol{R}}_{L} \boldsymbol{e}_{1}$. This can be obtained with $\boldsymbol{R}_{L}=\boldsymbol{R}_{L}(k, \phi)=$ $\overline{\boldsymbol{R}}_{L} \boldsymbol{R}_{\boldsymbol{z}_{L}}(k \pi) \boldsymbol{R}_{\boldsymbol{x}_{L}}(\phi)$, where $k=0,1, \phi \in[0,2 \pi]$, and $\boldsymbol{R}_{\boldsymbol{z}_{L}}(\cdot)$ and $\boldsymbol{R}_{\boldsymbol{x}_{L}}(\cdot)$ are the rotations about $\boldsymbol{z}_{L}$ and $\boldsymbol{x}_{L}$, respectively. Considering that ${ }^{L} \boldsymbol{b}_{1}$ is parallel to $\boldsymbol{x}_{L}$ we have that $\boldsymbol{R}_{\boldsymbol{z}_{L}}(k \pi) \boldsymbol{R}_{\boldsymbol{x}_{L}}(\phi)^{L} \boldsymbol{b}_{1}$ is either equal to ${ }^{L} \boldsymbol{b}_{1}$ if $k=0$ or to $-{ }^{L} \boldsymbol{b}_{1}$ if $k=1$. Therefore, using (14), we obtain either $\boldsymbol{p}_{L}=\overline{\boldsymbol{p}}_{L}$ if $k=0$ or $p_{L}=\bar{p}_{L}+2 b_{1}$ if $k=1$. Fig. 4 a provides a simplified representations of the two different sets of equilibria for $k=0$ and $k=1$, formally defined as follows:

- $\mathscr{Q}^{+}\left(t_{L}, \overline{\boldsymbol{q}}_{L}\right)=\left\{\boldsymbol{q} \in \mathscr{Q}\left(t_{L}, \overline{\boldsymbol{q}}_{L}\right) \mid \boldsymbol{R}_{L}=\boldsymbol{R}_{L}(0, \boldsymbol{\phi}) \forall \boldsymbol{\phi}\right\}$,

- $\mathscr{Q}^{-}\left(t_{L}, \overline{\boldsymbol{q}}_{L}\right)=\left\{\boldsymbol{q} \in \mathscr{Q}\left(t_{L}, \overline{\boldsymbol{q}}_{L}\right) \mid \boldsymbol{R}_{L}=\boldsymbol{R}_{L}(1, \boldsymbol{\phi}) \forall \boldsymbol{\phi}\right\}$.

Notice that $\mathscr{Q}\left(0, \overline{\boldsymbol{q}}_{L}\right)$ is parametrized by an element in $S O(3)$ (any $\boldsymbol{R}_{L} \in S O(3)$ is allowed), while $\mathscr{Q}^{+}\left(t_{L}, \overline{\boldsymbol{q}}_{L}\right)$ and $\mathscr{Q}^{-}\left(t_{L}, \overline{\boldsymbol{q}}_{L}\right)$, for $t_{L} \neq 0$, are parametrized by an element in 
$S O(1)\left(\boldsymbol{R}_{L}(0, \phi)\right.$ and $\boldsymbol{R}_{L}(1, \phi)$, for any $\phi \in[0,2 \pi]$, respectively). For all $t_{L}$, the load rotation about $\boldsymbol{x}_{L}$ is arbitrary because the robots can not apply any torque along $\boldsymbol{x}_{L}$, so the corresponding rotation results uncontrollable.

We can conclude that choosing $t_{L}=0$ (equilibrium with vertical cables) every orientation of the load is contained in the equilibrium set and the load equilibrium positions are free to move on a sphere of radius $\left\|{ }^{L} \boldsymbol{b}_{1}\right\|$ centered on $B_{1}$. Contrarily, $t_{L} \neq 0$ is a much better choice. In this case, a part from the rotation about the $x_{L}$ axis, there are only two distinct equilibria, and one is exactly $\boldsymbol{q}_{L}=\overline{\boldsymbol{q}}_{L}$, as expected. For the other one the load orientation is parallel to the one in $\bar{q}_{L}$ but its position is reflected w.r.t. $B_{1}$ (see Fig. 4 a for an example).

\section{Stability of The EQuilibria}

In this section we shall analyze the stability of the equilibria discovered in Sec. III-A. Firstly we define $\boldsymbol{x}=(\boldsymbol{q}, \boldsymbol{v})$ as the state of the system, $\overline{\boldsymbol{x}}=(\overline{\boldsymbol{q}}, \mathbf{0})$ the desired equilibrium state, and the following sets (subspaces of the state space):

- $\mathscr{X}\left(t_{L}, \overline{\boldsymbol{q}}_{L}\right)=\left\{\boldsymbol{x}: \boldsymbol{q} \in \mathscr{Q}\left(t_{L}, \overline{\boldsymbol{q}}_{L}\right), \boldsymbol{v}=\mathbf{0}\right\}$,

- $\mathscr{X}\left(0, \overline{\boldsymbol{q}}_{L}\right)=\left\{\boldsymbol{x}: \boldsymbol{q} \in \mathscr{Q}\left(0, \overline{\boldsymbol{q}}_{L}\right), \boldsymbol{v}=\mathbf{0}\right\}$,

- $\mathscr{X}^{+}\left(t_{L}, \overline{\boldsymbol{q}}_{L}\right)=\left\{\boldsymbol{x}: \boldsymbol{q} \in \mathscr{Q}^{+}\left(t_{L}, \overline{\boldsymbol{q}}_{L}\right), \boldsymbol{v}=\mathbf{0}\right\}$,

- $\mathscr{X}^{-}\left(t_{L}, \overline{\boldsymbol{q}}_{L}\right)=\left\{\boldsymbol{x}: \boldsymbol{q} \in \mathscr{Q}^{-}\left(t_{L}, \overline{\boldsymbol{q}}_{L}\right), \boldsymbol{v}=\mathbf{0}\right\}$.

Theorem 3. Let us consider a desired load configuration $\overline{\boldsymbol{q}}_{L}$. For the system (4) let the constant forcing input $\pi_{A}$ be chosen in $\Pi_{A}\left(\overline{\boldsymbol{q}}_{L}\right)$ corresponding to a certain internal force $t_{L}$. Then $\boldsymbol{x}$ belonging to:

- $\mathscr{X}^{+}\left(t_{L}, \bar{q}_{L}\right)$ is locally asymptotically stable if $t_{L}>0$;

- $\mathscr{X}^{-}\left(t_{L}, \overline{\boldsymbol{q}}_{L}\right)$ is unstable if $t_{L}>0$;

- $\mathscr{X}\left(0, \bar{q}_{L}\right)$ is locally asymptotically stable;

- $\mathscr{X}^{+}\left(t_{L}, \overline{\boldsymbol{q}}_{L}\right)$ is unstable if $t_{L}<0$;

- $\mathscr{X}^{-}\left(t_{L}, \bar{q}_{L}\right)$ is locally asymptotically stable if $t_{L}<0$.

Proof. Let us consider the following Lyapunov candidate:

$$
\begin{aligned}
V(\boldsymbol{x})= & \frac{1}{2}\left(\boldsymbol{v}_{R}^{\top} \boldsymbol{M}_{A} \boldsymbol{v}_{R}+\boldsymbol{e}_{R}^{\top} \boldsymbol{K}_{A} \boldsymbol{e}_{R}+\boldsymbol{v}_{L}^{\top} \boldsymbol{M}_{L} \boldsymbol{v}_{L}+\right. \\
& \left.+k_{1}\left(\left\|\boldsymbol{l}_{1}\right\|-l_{01}\right)^{2}+k_{2}\left(\left\|\boldsymbol{l}_{2}\right\|-l_{02}\right)^{2}\right)-\boldsymbol{l}_{1}^{\top} \overline{\boldsymbol{f}}_{1}+ \\
& -\boldsymbol{l}_{2}^{\top} \overline{\boldsymbol{f}}_{2}+t_{L}\left(1-\left(\overline{\boldsymbol{R}}_{L} \boldsymbol{e}_{1}\right)^{\top} \boldsymbol{R}_{L} \boldsymbol{e}_{1}\right)+V_{0},
\end{aligned}
$$

where $V_{0} \in \mathbb{R}_{\geq 0}$ and $\boldsymbol{e}_{R}=\overline{\boldsymbol{p}}_{R 1}-\boldsymbol{p}_{R 1}$. For an opportune choice of $V_{0}, V(\boldsymbol{x})$ is a positive definite, continuously differentiable function in the domain of interest for which we have that $\boldsymbol{x}_{\text {min }}=\operatorname{argmin}_{\boldsymbol{x}} V(\boldsymbol{x})$ is such that $\boldsymbol{x}_{\text {min }} \in \mathscr{X}\left(0, \overline{\boldsymbol{q}}_{L}\right)$ and $\boldsymbol{x}_{\text {min }} \in \mathscr{X}^{+}\left(t_{L}, \overline{\boldsymbol{q}}_{L}\right)$ for $t_{L}>0$. The complete proof is provided in technical report in the multimedia materials. In particular, if $t_{L} \geq 0$, we can choose the term $V_{0}$ such that $V(\boldsymbol{x}) \geq 0$ and $V(\overline{\boldsymbol{x}})=0$. Notice that $V(\boldsymbol{x})=0$ for all $\boldsymbol{x} \in \mathscr{X}\left(0, \overline{\boldsymbol{q}}_{L}\right)$ and $\boldsymbol{x} \in \mathscr{X}^{+}\left(t_{L}, \overline{\boldsymbol{q}}_{L}\right)$ for $t_{L}>0$.

Computing the time derivative of (18) and replacing (4), (1) and (8) we obtain $\dot{V}=-\boldsymbol{v}_{R}^{\top} \boldsymbol{B}_{A} \boldsymbol{v}_{R}-\boldsymbol{\omega}_{L}^{\top} \boldsymbol{B}_{L} \boldsymbol{\omega}_{L}$ that is clearly negative semidefinite. In particular $\dot{V}(\boldsymbol{x})=0$ for all $\boldsymbol{x} \in \mathscr{E}\left\{\boldsymbol{x}: \boldsymbol{v}_{R}=\mathbf{0}, \boldsymbol{\omega}_{L}=\mathbf{0}\right\}$

Since $\dot{V}$ is only negative semidefinite, to prove the asymptotic stability we rely on the LaSalle's invariance principle [17]. Let us define a positively invariant set $\Omega_{\alpha}=\{\boldsymbol{x}$ :
$V(\boldsymbol{x}) \leq \alpha$ with $\left.\alpha \in \mathbb{R}_{>0}\right\}$. By construction $\Omega_{\alpha}$ is compact since (18) is radially unbounded and $\Omega_{0}$ is compact $\left(\Omega_{0}=\right.$ $\mathscr{X}\left(0, \overline{\boldsymbol{q}}_{L}\right)$ and $\Omega_{0}=\mathscr{X}^{+}\left(t_{L}, \overline{\boldsymbol{q}}_{L}\right)$ for $t_{L}=0$ and $t_{L}>0$, respectively, are both compact sets). Then we need to find the largest invariant set $\mathscr{M}$ in $\mathscr{E}=\left\{\boldsymbol{x} \in \Omega_{\alpha} \mid \dot{V}(\boldsymbol{x})=0\right\}$. A trajectory $\boldsymbol{x}(t)$ belongs identically to $\mathscr{E}$ if $\dot{V}(\boldsymbol{x}(t)) \equiv$ $0 \Leftrightarrow \boldsymbol{v}_{R}(t) \equiv \mathbf{0}$ and $\boldsymbol{\omega}_{L}(t) \equiv \mathbf{0} \Leftrightarrow m\left(\boldsymbol{q}(t), \mathbf{0}, \boldsymbol{\pi}_{A}\right)=\mathbf{0}$ for all $t \in \mathbb{R}_{>0}$. Therefore $\boldsymbol{x}$ has to be an equilibrium, and from Theorem 2 we have that $\dot{V}(\boldsymbol{x}(t)) \equiv 0 \Leftrightarrow \boldsymbol{x}(t) \in \mathscr{X}\left(t_{L}, \overline{\boldsymbol{q}}_{L}\right)$. Thus we obtain $\mathscr{M}=\Omega_{\alpha} \cap \mathscr{X}\left(t_{L}, \bar{q}_{L}\right)$.

For $t_{L}>0$, it is easy to see that for a sufficiently small $\alpha, \mathscr{X}^{+}\left(t_{L}, \overline{\boldsymbol{q}}_{L}\right) \subseteq \Omega_{\alpha}$ but $\mathscr{X}^{-}\left(t_{L}, \overline{\boldsymbol{q}}_{L}\right) \cap \Omega_{\alpha}=\varnothing$. This because $V(\boldsymbol{x})=0$ for $\boldsymbol{x} \in \mathscr{X}^{+}\left(t_{L}, \overline{\boldsymbol{q}}_{L}\right)$, while $V(\boldsymbol{x})>0$ for $\boldsymbol{x} \in \mathscr{X}^{-}\left(t_{L}, \overline{\boldsymbol{q}}_{L}\right)$. Indeed, in (18), for $\boldsymbol{x} \in \mathscr{X}^{-}\left(t_{L}, \overline{\boldsymbol{q}}_{L}\right)$, the term $t_{L}\left(1-\left(\overline{\boldsymbol{R}}_{L} \boldsymbol{e}_{1}\right)^{\top} \boldsymbol{R}_{L} \boldsymbol{e}_{1}\right)=2 t_{L}>0$. Therefore $\mathscr{M}=$ $\mathscr{X}^{+}\left(t_{L}, \overline{\boldsymbol{q}}_{L}\right)$. All conditions of LaSalle's principle are satisfied and $\mathscr{X}^{+}\left(t_{L}, \overline{\boldsymbol{q}}_{L}\right)$ is locally asymptotically stable.

On the other hand, for $t_{L}=0$ we have that $\mathscr{X}\left(t_{L}, \overline{\boldsymbol{q}}_{L}\right) \subseteq \Omega_{\alpha}$ for every sufficiently small $\alpha$. Therefore $\mathscr{M}=\mathscr{X}\left(t_{L}, \overline{\boldsymbol{q}}_{L}\right)$ and, as before, we can conclude that $\mathscr{X}\left(t_{L}, \overline{\boldsymbol{q}}_{L}\right)$ is locally asymptotically stable for the LaSalle's invariance principle.

Now, let us investigate the stability for $t_{L}<0$. As before, with an opportune choice of $V_{0}$, we have that $V(\boldsymbol{x})=0$ for $\boldsymbol{x} \in \mathscr{X}^{+}\left(t_{L}, \overline{\boldsymbol{q}}_{L}\right)$. However $\mathscr{X}^{+}\left(t_{L}, \overline{\boldsymbol{q}}_{L}\right)$ is a set of accumulation for the points where $V(\boldsymbol{x})<0$. Indeed, consider $\boldsymbol{v}=\mathbf{0}, \boldsymbol{p}_{R 1}=\overline{\boldsymbol{p}}_{R 1}, \boldsymbol{R}_{L}$ such that $\left(\overline{\boldsymbol{R}}_{L} \boldsymbol{e}_{1}\right)^{\top} \boldsymbol{R}_{L} \boldsymbol{e}_{1}=1-\varepsilon$, with $\varepsilon>0$ arbitrarily small, $\boldsymbol{p}_{L}$ and $\boldsymbol{p}_{R 2}$ as in (14). Under this conditions, we have that $V(\boldsymbol{x})=t_{L}\left(1-\left(\overline{\boldsymbol{R}}_{L} \boldsymbol{e}_{1}\right)^{\top} \boldsymbol{R}_{L} \boldsymbol{e}_{1}\right)=$ $t_{L} \varepsilon<0$. Then, $\dot{V}(\boldsymbol{x})<0$ in a neighborhood of $\mathscr{X}^{+}\left(t_{L}, \overline{\boldsymbol{q}}_{L}\right)$. All conditions of Chetaev's theorem [17] are satisfied, and we can conclude that $\mathscr{X}^{+}\left(t_{L}, \overline{\boldsymbol{q}}_{L}\right)$ is an unstable set.

Finally, to study the stability of $\mathscr{X}^{-}\left(t_{L}, \overline{\boldsymbol{q}}_{L}\right)$ for $t_{L} \neq 0$, let us consider a desired load configuration $\overline{\boldsymbol{q}}_{L}^{\prime}=\left(\overline{\boldsymbol{p}}_{L}^{\prime}, \overline{\boldsymbol{R}}_{L}^{\prime}\right)$ such that $\overline{\boldsymbol{p}}_{L}^{\prime}=\boldsymbol{p}_{L}^{\prime}+2 \overline{\boldsymbol{R}}_{L} \boldsymbol{e}_{1}$ and $\overline{\boldsymbol{R}}_{L}^{\prime}=\boldsymbol{R}_{L}(1, \phi)$ for a certain $\phi$. Then we choose $\pi_{A}^{\prime} \in \Pi_{A}\left(\overline{\boldsymbol{q}}_{L}^{\prime}\right)$ with $t_{L}^{\prime}=-t_{L}$. For the reasoning in Sec. III-A, we have that $\mathscr{X}^{+}\left(t_{L}^{\prime}, \overline{\boldsymbol{q}}_{L}^{\prime}\right)=$ $\mathscr{X}^{-}\left(t_{L}, \overline{\boldsymbol{q}}_{L}\right)$. Furthermore, for the previous results, if $t_{L}>0$, $t_{L}^{\prime}<0$ and $\mathscr{X}^{+}\left(t_{L}^{\prime}, \overline{\boldsymbol{q}}_{L}^{\prime}\right)$ is unstable. Therefore, $\mathscr{X}^{-}\left(t_{L}, \overline{\boldsymbol{q}}_{L}\right)$ is unstable too. A similar reasoning can be done to prove that $\mathscr{X}^{-}\left(t_{L}, \overline{\boldsymbol{q}}_{L}\right)$ is locally asymptotically stable for $t_{L}<0$.

\section{Passivity and Stability of Manipulation}

Theorem 3 characterizes the stability of all the possible static equilibria given a certain constant forcing input. In particular, it shows that one has to choose $t_{L}>0$ and $\pi_{A} \in$ $\Pi_{A}\left(\overline{\boldsymbol{q}}_{L}\right)$ to let the system asymptotically converge to a desired load configuration. On the contrary, one must avoid $t_{L}=0$ because the control of the load attitude and its position is not possible. Notice that this last case is the most used in the literature, where the attempt is made to keep the cables always vertical, i.e., with no internal forces.

Let us now show how one can exploit the input $\pi_{A 1}$ in order to move the load between two distinct positions. From (6)-(8) and from the fact that $\boldsymbol{K}_{A 2}=\mathbf{0}$, it descends that

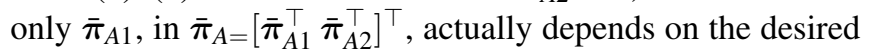
load position $\overline{\boldsymbol{p}}_{L}$. This makes robot 1 able to steer alone the load position without communicating with robot 2 . This is 
done by first plugging a new desired position $\bar{p}_{L}^{\prime}$ in (6) thus computing a new $\bar{p}_{R 1}^{\prime}$, and then plugging $\bar{p}_{R 1}^{\prime}$ in (7) in order to compute the new constant forcing input $\bar{\pi}_{A 1}^{\prime}$. However, one may want to minimize the transient phases generated by a piecewise constant forcing input. It is sufficient to design $\pi_{A 1}$ as

$$
\boldsymbol{\pi}_{A 1}(t)=\overline{\boldsymbol{\pi}}_{A 1}+\boldsymbol{u}_{A 1}(t),
$$

where $\boldsymbol{u}_{A 1}(t)$ is a smooth function such that $\boldsymbol{\pi}_{A 1}(0)=\overline{\boldsymbol{\pi}}_{A 1}$ and $\pi_{A 1}\left(t_{f}\right)=\bar{\pi}_{A 1}^{\prime}$ for $t_{f} \in \mathbb{R}_{>0}$.

To ensure that the system remains stable when the input is time-varying, we shall prove that the system is output-strictly passive w.r.t. the input-output pair $(\boldsymbol{u}, \boldsymbol{y})=\left(\boldsymbol{u}_{A}, \boldsymbol{v}_{R}\right)$.

Theorem 4. If $\boldsymbol{\pi}_{A}$ is defined as in (19) for a certain $\overline{\boldsymbol{q}}$ and $\overline{\boldsymbol{q}}^{\prime}$ with $t_{L} \geq 0$, then system (4) is output-strictly passive w.r.t. the storage function (18) and the input-output pair $(\boldsymbol{u}, \boldsymbol{y})=$ $\left(\boldsymbol{u}_{A}, \boldsymbol{v}_{R}\right)$.

Proof. In the proof of Theorem 3 we already shown that (18) is a continuously differentiable positive definite function for $t_{L} \geq 0$, properly choosing $V_{0}$. Furthermore, replacing (19) into (3), and differentiating (18) we obtain

$$
\begin{aligned}
\dot{V} & =-\boldsymbol{v}_{R}^{\top} \boldsymbol{B}_{A} \boldsymbol{v}_{R}+\boldsymbol{v}_{R}^{\top} \boldsymbol{u}_{A}-\boldsymbol{\omega}_{L}^{\top} \boldsymbol{B}_{L} \boldsymbol{\omega}_{L} \\
& \leq \boldsymbol{u}^{\top} \boldsymbol{y}-\boldsymbol{y}^{\top} \boldsymbol{B}_{A} \boldsymbol{y}=\boldsymbol{u}^{\top} \boldsymbol{y}-\boldsymbol{y}^{\top} \boldsymbol{\Phi}(\boldsymbol{y}),
\end{aligned}
$$

with $\boldsymbol{y}^{\top} \boldsymbol{\Phi}(\boldsymbol{y})>0 \forall \boldsymbol{y} \neq \mathbf{0}$. Therefore, system (4) is outputstrictly passive [17].

Thanks to the passivity of the system we can say that for a bounded input provided to the master, the energy of the system remains bounded too, and in particular it stabilizes to a new constant value as soon as $\boldsymbol{u}_{A 1}$ becomes constant again. This means that while moving the master, the overall state of the system will remain bounded, and will converge to another specific equilibrium configuration when the master input becomes constant. Furthermore, it is well known that passivity is a robust property, especially w.r.t. model uncertainties. In particular, choosing $\pi_{A} \in \Pi_{A}\left(\overline{\boldsymbol{q}}_{L}\right)$ for a given $\overline{\boldsymbol{q}}$, the system remains asymptotically stable even in the presence of some parameter uncertainties, but it will converge to a $\overline{\boldsymbol{q}}^{\prime}$ that is slightly different from $\overline{\boldsymbol{q}}$.

Remark 4. Once the desired load pose is decided and the value of $t_{L}$ is chosen, one can compute the control input $\pi_{A}$ and send it to the robots. Afterwards, if $t_{L}>0$ the robots will steer the load to the desired configuration preserving the stability and without the need of sending data to each other. The cooperative task is performed exploiting the implicit communication through the forces that the robots exchange and feel from the cables and the object.

\section{NUmerical VALIDATION}

In this section we shall describe the results of several numerical simulations validating the proposed method and all the presented theoretical concepts and results.

For the simulation we considered a quadrotor-like vehicle with its proper nonlinear dynamics together with a geometric position controller, even though, our method can be applied to more general flying vehicles. System and control parameters are reported in Tab. I. Notice the smaller apparent inertia of the slave, chosen to make it more sensitive to external forces.

Let us consider the desired equilibrium $\overline{\boldsymbol{q}}=\left(\overline{\boldsymbol{p}}_{L}, \overline{\boldsymbol{R}}_{L}\right)$, whose value are in Tab. I, where $(\bar{\phi}, \bar{\theta}, \bar{\psi})$ are the Euler angles that parametrize $\overline{\boldsymbol{R}}_{L}$. We performed several simulations with $\pi_{A} \in \Pi_{A}\left(\bar{q}_{L}\right)$ computed as in (6) for the cases: 1) $t_{L 1}=1.5[\mathrm{~N}]>0$, 2) $\left.t_{L 2}=0[\mathrm{~N}], 3\right) t_{L 3}=-1[\mathrm{~N}]<0$.

To test the stability of the equilibria, we initialized the system in different initial configurations and we let it evolve. Figure 6 shows the position and orientation error for the three $t_{L}$ and several different initial conditions. 1) For $t_{L}=t_{L 1}$, the system always converges to a state belonging to $\mathscr{X}^{+}\left(t_{L}, \overline{\boldsymbol{q}}_{L}\right)$, independently from the initial state, validating the asymptotic stability of $\mathscr{X}^{+}\left(t_{L}, \overline{\boldsymbol{q}}_{L}\right)$ when $t_{L}>0$. 2) For $t_{L 2}$, the system final state belongs to $\mathscr{X}\left(0, \bar{q}_{L}\right)$. The particular final attitude of the load depends on the initial state. 3) For $t_{L 3}$, the system never converges to $\mathscr{X}^{+}\left(t_{L}, \overline{\boldsymbol{q}}_{L}\right)$ even with a very close initial configuration. This is due to the instability of $\mathscr{X}^{+}\left(t_{L}, \overline{\boldsymbol{q}}_{L}\right)$ when $t_{L}<0$. Fig. 5 shows the evolution of the system starting from two different initial states for the three cases.

In another set of simulations, shown in detail in the attached technical report, the master input $\pi_{A 1}(t)$ is chosen as in (19) to bring the load in $\bar{p}_{L}^{\prime}=\left[\begin{array}{lll}4.5 & 4.5 & 5\end{array}\right]^{\top}[\mathrm{m}]$. We observed that, as expected, for both $t_{L}=t_{L 1}$ and $t_{L}=t_{L 2}$ the system remains stable during the master maneuver. Once the input becomes constant, the master stops and the system converges to $\overline{\boldsymbol{q}}$ for $t_{L}=t_{L 1}$. For $t_{L}=t_{L 2}$, the final load attitude depends on the particular motion, and it is in general different from $\overline{\boldsymbol{q}}$.

Additional simulations in non-ideal conditions are provided in the attached technical report. The results show that thanks to the passivity of the system, the latter is very robust to the considered non-idealities. Some representative simulations are available in the attached video too.

\section{CONCLUSIONS}

This work deals with the decentralized cooperative manipulation of a cable-suspended load performed by two aerial vehicles. The proposed master-slave architecture exploits an admittance controller in order to coordinate the robots with implicit communication only, exploiting the cable forces. The passivity of the system has been proven, and the stability of

\begin{tabular}{|c|c|c|c|c|c|}
\hline \multicolumn{3}{|c|}{ System Parameters } & \multicolumn{3}{|c|}{ Controller Gains } \\
\hline & $i=1$ & $i=2$ & & $i=1$ & $i=2$ \\
\hline \multirow{5}{*}{$\begin{array}{c}m_{R i}[\mathrm{Kg}] \\
J_{R i}\left[\mathrm{Kg} \cdot \mathrm{m}^{2}\right] \\
l_{0 i}[\mathrm{~m}] \\
k_{i}[\mathrm{~N} / \mathrm{m}] \\
{ }^{L} \boldsymbol{b}_{i}[\mathrm{~m}]\end{array}$} & \multirow{5}{*}{$\begin{array}{c}1.02 \\
0.015 \boldsymbol{I}_{3} \\
1 \\
20 \\
{\left[\begin{array}{lll}0.433 & 0 & 0\end{array}\right]}\end{array}$} & \multirow{5}{*}{$\begin{array}{c}0.993 \\
0.015 \mathbf{I}_{3} \\
1 \\
20 \\
{[-0.43300}\end{array}$} & $M_{A i}$ & $3 I_{3}$ & $0.5 \boldsymbol{I}_{3}$ \\
\hline & & & $\boldsymbol{B}_{A i}$ & $18 \boldsymbol{I}_{3}$ & $1.3 \boldsymbol{I}_{3}$ \\
\hline & & & $\boldsymbol{K}_{A i}$ & $15 \boldsymbol{I}_{3}$ & $\mathbf{0}$ \\
\hline & & & \multicolumn{3}{|c|}{ Desired Load Pose } \\
\hline & & & \multirow{2}{*}{\multicolumn{3}{|c|}{ 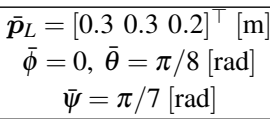 }} \\
\hline \multicolumn{3}{|c|}{$\begin{array}{c}m_{L}=0.900[\mathrm{Kg}], \boldsymbol{J}_{L x}=0.112\left[\mathrm{Kg} \cdot \mathrm{m}^{2}\right] \\
\boldsymbol{J}_{L y}=5.681, \boldsymbol{J}_{L z}=5.681\left[\mathrm{Kg} \cdot \mathrm{m}^{2}\right]\end{array}$} & & & \\
\hline
\end{tabular}
the static equilibria has been studied highlighting the crucial role of the internal force. In particular, contrarily from what

TABLE I: Parameters used in the simulations. 


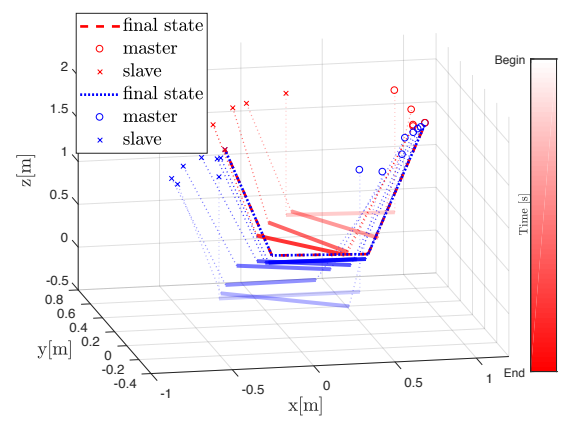

(a) $t_{L}=t_{L 1}>0$

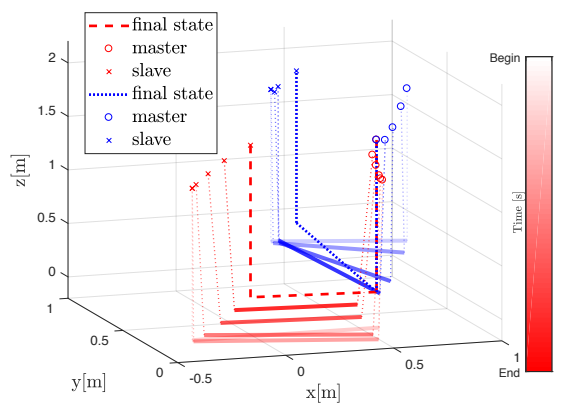

(b) $t_{L}=t_{L 2}=0$

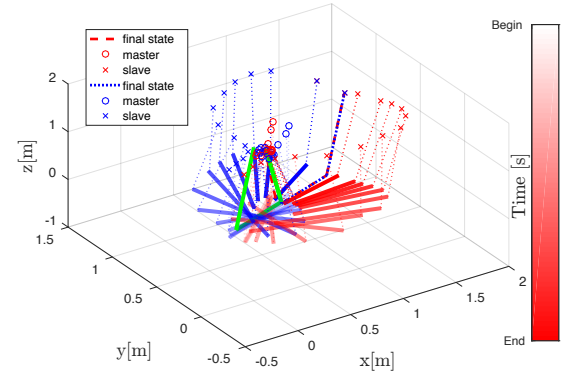

(c) $t_{L}=t_{L 3}<0$

Fig. 5: Each figure shows the evolution of the system from two different initial conditions (one is shown in red and the other in blue). The two evolutions are represented as a sequence of images discriminated by the brightness of the color that represents the time (from bright/start to dark/end). The load is represented as a tick solid line, the cables as thin dashed lines, the master robot as a circle and the slave robot as a cross.
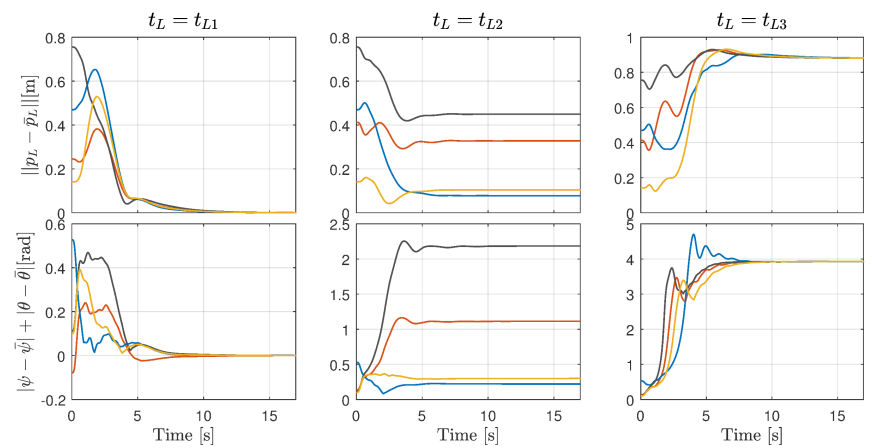

Fig. 6: Convergence to the desired load configuration for cases 1) 2) and 3). In particular the first and second rows show the position and the attitude errors, respectively, for four different initial conditions (different colors) and for the three different internal force values (columns). The attitude error is computed as the sum of pitch and yaw errors. The roll error is not considered since it is not controllable.

it is normally done in the literature (zero internal force), it is advisable to choose a positive internal force to control both position and orientation of the beam. In the future it would be interesting to test the method on real platforms and to extend the analysis to general loads or to agile motions. An extension to a more generic load attached to $N$ robots could be very interesting too.

\section{REFERENCES}

[1] M. Tognon, B. Yüksel, G. Buondonno, and A. Franchi, "Dynamic decentralized control for protocentric aerial manipulators," in 2017 IEEE Int. Conf. on Robotics and Automation, Singapore, May 2017, pp. 6375-6380.

[2] M. Tognon, A. Testa, E. Rossi, and A. Franchi, "Takeoff and landing on slopes via inclined hovering with a tethered aerial robot," in 2016 IEEE/RSJ Int. Conf. on Intelligent Robots and Systems, Daejeon, South Korea, Oct. 2016, pp. 1702-1707.

[3] M. Tognon and A. Franchi, "Dynamics, control, and estimation for aerial robots tethered by cables or bars," IEEE Trans. on Robotics, vol. 33, no. 4, pp. 834-845, 2017.

[4] I. Maza, K. Kondak, M. Bernard, and A. Ollero, "Multi-UAV cooperation and control for load transportation and deployment," Journal of Intelligent \& Robotics Systems, vol. 57, no. 1-4, pp. 417-449, 2010.

[5] H.-N. Nguyen, S. Park, and D. J. Lee, "Aerial tool operation system using quadrotors as rotating thrust generators," in 2015 IEEE/RSJ Int.
Conf. on Intelligent Robots and Systems, Hamburg, Germany, Oct. 2015, pp. 1285-1291.

[6] R. Ritz and R. D'Andrea, "Carrying a flexible payload with multiple flying vehicles," in 2013 IEEE/RSJ Int. Conf. on Intelligent Robots and Systems, 2013, pp. 3465-3471.

[7] F. Caccavale, G. Giglio, G. Muscio, and F. Pierri, "Cooperative impedance control for multiple uavs with a robotic arm," in 2015 IEEE/RSJ Int. Conf. on Intelligent Robots and Systems, 2015, pp. 2366-2371.

[8] K. Sreenath and V. Kumar, "Dynamics, control and planning for cooperative manipulation of payloads suspended by cables from multiple quadrotor robots," in Robotics: Science and Systems, Berlin, Germany, June 2013.

[9] C. Masone, H. H. Bülthoff, and P. Stegagno, "Cooperative transportation of a payload using quadrotors: A reconfigurable cable-driven parallel robot," in 2016 IEEE/RSJ Int. Conf. on Intelligent Robots and Systems, Oct 2016, pp. 1623-1630.

[10] M. Manubens, D. Devaurs, L. Ros, and J. Cortés, "Motion planning for 6-D manipulation with aerial towed-cable systems," in 2013 Robotics: Science and Systems, Berlin, Germany, May 2013.

[11] D. Mellinger, M. Shomin, N. Michael, and V.Kumar, "Cooperative grasping and transport using multiple quadrotors," in Int. Symp. on Distributed Autonomous Robotic Systems, 2013, pp. 545-558.

[12] Z. Wang and M. Schwager, "Force-amplifying n-robot transport system (force-ants) for cooperative planar manipulation without communication," The International Journal of Robotics Research, vol. 35, no. 13, pp. 1564-1586, 2016.

[13] A. Tagliabue, M. Kamel, S. Verling, R. Siegwart, and J. Nieto, "Collaborative transportation using MAVs via passive force control," in 2017 IEEE Int. Conf. on Robotics and Automation, Singapore, 2016, pp. 5766-5773.

[14] M. Gassner, T. Cieslewski, and D. Scaramuzza, "Dynamic collaboration without communication: Vision-based cable-suspended load transport with two quadrotors," in 2017 IEEE Int. Conf. on Robotics and Automation, Singapore, 2017.

[15] M. Ryll, D. Bicego, and A. Franchi, "Modeling and control of FASTHex: a fully-actuated by synchronized-tilting hexarotor," in 2016 IEEE/RSJ Int. Conf. on Intelligent Robots and Systems, Daejeon, South Korea, Oct. 2016, pp. 1689-1694.

[16] M. Ryll, G. Muscio, F. Pierri, E. Cataldi, G. Antonelli, F. Caccavale, and A. Franchi, "6D physical interaction with a fully actuated aerial robot," in 2017 IEEE Int. Conf. on Robotics and Automation, Singapore, May 2017, pp. 5190-5195.

[17] H. K. Khalil, Nonlinear Systems, 3rd ed. Prentice Hall, 2001. 


\title{
Additional Analysis and Simulations for Communication-Less Cooperative Aerial Manipulation
}

\author{
Technical report of: \\ "Aerial Co-Manipulation with Cables: The Role of Internal Force for Equilibria, Stability, and Passivity" \\ IEEE Robotics and Automation Letters, Special Issue on Aerial Manipulation
}

Marco Tognon ${ }^{1}$, Chiara Gabellieri ${ }^{1 \dagger}$, Lucia Pallottino ${ }^{2}$, and Antonio Franchi ${ }^{1}$

\begin{abstract}
This document is a technical attachment to [1] as an extension of the theoretical analysis and of the numerical validation part. Here we present additional plots and additional simulations in presence of non-ideal conditions as noise and parameter variations. A thorough validation of the robustness of the proposed method against the aforementioned non-idealities is also conducted.
\end{abstract}

\section{How to Cite This Work}

This technical report is accompanying our IEEE Robotics and Automation Letters paper [1]. If you wish to reference this work, please cite this paper as follows:

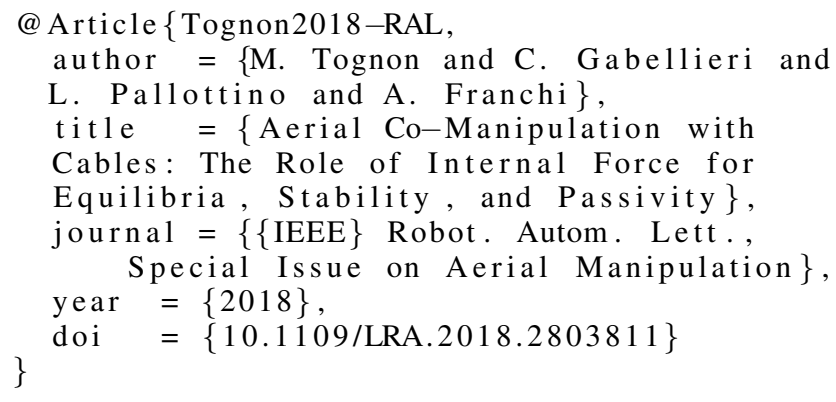

\section{INTRODUCTION}

In Sec. III we integrate the Lyapunov based stability analysis of Sec. IV of the manuscript. In particular we shall prove that the Lyapunov function (18) of the manuscript is positive definite and has global minima in a particular set of interest.

Then, in the following sections, we shall describe several additional simulations validating the proposed method and all the theoretical concepts and results presented in [1]. The parameter of the simulated system are the one reported in [1].

\footnotetext{
$\dagger$ The first two authors have equally collaborated to the manuscript, and can both be considered as first author.

${ }^{1}$ LAAS-CNRS, Université de Toulouse, CNRS, Toulouse, France, antonio.franchillaas.fr, marco.tognon@laas.fr, cgabellieri@laas.fr

${ }^{2}$ Centro di ricerca E. Piaggio, Università di Pisa, Largo Lucio Lazzarino 1, 56122,Pisa, Italy lucia.pallottino@unipi.it

This work has been funded by the European Union's Horizon 2020 research and innovation program under grant agreement No 644271 AEROARMS.
}

\section{LYAPUNOV FUNCTION CHARACTERIZATION}

In the following proposition we analyze in details the Lyapunov candidate:

$$
\begin{aligned}
V(\boldsymbol{x})= & \frac{1}{2}\left(\boldsymbol{v}_{R}^{\top} \boldsymbol{M}_{A} \boldsymbol{v}_{R}+\boldsymbol{e}_{R}^{\top} \boldsymbol{K}_{A} \boldsymbol{e}_{R}+\boldsymbol{v}_{L}^{\top} \boldsymbol{M}_{L} \boldsymbol{v}_{L}+\right. \\
& \left.+k_{1}\left(\left\|\boldsymbol{l}_{1}\right\|-l_{01}\right)^{2}+k_{2}\left(\left\|\boldsymbol{l}_{2}\right\|-l_{02}\right)^{2}\right)-\boldsymbol{l}_{1}^{\top} \overline{\boldsymbol{f}}_{1}+ \\
& -\boldsymbol{l}_{2}^{\top} \overline{\boldsymbol{f}}_{2}+t_{L}\left(1-\left(\overline{\boldsymbol{R}}_{L} \boldsymbol{e}_{1}\right)^{\top} \boldsymbol{R}_{L} \boldsymbol{e}_{1}\right)+V_{0},
\end{aligned}
$$

used in Sec. IV and Sec. V of the manuscript, for the proofs of stability and passivity of the system.

Proposition 1. Considering the Lyapunov function (1), we have that:

- $\boldsymbol{x}_{\min }=\operatorname{argmin}_{\boldsymbol{x}} V(\boldsymbol{x})$ is such that $\boldsymbol{x}_{\min } \in \mathscr{X}\left(0, \overline{\boldsymbol{q}}_{L}\right)$ and $\boldsymbol{x}_{\min } \in \mathscr{X}^{+}\left(t_{L}, \overline{\boldsymbol{q}}_{L}\right)$ for $t_{L}>0$;

- $V(\boldsymbol{x})$ is positive definite for an opportune choice of $V_{0}$.

Proof. We divide (1) into three parts such that

$$
V(\boldsymbol{x})=\bar{V}(\boldsymbol{x})+V_{1}(\boldsymbol{x})+V_{2}(\boldsymbol{x}),
$$

where

$$
\begin{aligned}
\bar{V}(\boldsymbol{x})= & \frac{1}{2}\left(\boldsymbol{v}_{R}^{\top} \boldsymbol{M}_{A} \boldsymbol{v}_{R}+\boldsymbol{e}_{R}^{\top} \boldsymbol{K}_{A} \boldsymbol{e}_{R}+\boldsymbol{v}_{L}^{\top} \boldsymbol{M}_{L} \boldsymbol{v}_{L}+\right. \\
& +t_{L}\left(1-\left(\overline{\boldsymbol{R}}_{L} \boldsymbol{e}_{1}\right)^{\top} \boldsymbol{R}_{L} \boldsymbol{e}_{1}\right)+V_{0} \\
V_{i}(\boldsymbol{x})= & \frac{1}{2} k_{i}\left(\left\|\boldsymbol{l}_{i}\right\|-l_{0 i}\right)^{2}-\boldsymbol{l}_{i}^{\top} \overline{\boldsymbol{f}}_{i},
\end{aligned}
$$

for $i=1,2$.

We firstly show that the Lyapunov function is radially unbounded (also called coercive), i.e., $\lim _{\|\boldsymbol{x}\| \rightarrow \infty} V(\boldsymbol{x})=\infty$. Indeed, we have that clearly $\lim _{\|\boldsymbol{x}\| \rightarrow \infty} \bar{V}(\boldsymbol{x})=\infty$, while

$$
\begin{aligned}
& \lim _{\|\boldsymbol{x}\| \rightarrow \infty} V_{i}(\boldsymbol{x}) \\
& \lim _{\|\boldsymbol{x}\| \rightarrow \infty} \frac{1}{2} k_{i}\left(\left\|\boldsymbol{l}_{i}\right\|-l_{0 i}\right)^{2}-\boldsymbol{l}_{i}^{\top} \overline{\boldsymbol{f}}_{i}= \\
& \lim _{\|\boldsymbol{x}\| \rightarrow \infty} \frac{1}{2} k_{i}\left(\left\|\boldsymbol{l}_{i}\right\|^{2}+l_{0 i}^{2}\right)-k_{i}\left\|\boldsymbol{l}_{i}\right\| l_{0 i}-\boldsymbol{l}_{i}^{\top} \overline{\boldsymbol{f}}_{i} \geq \\
& \lim _{\|\boldsymbol{x}\| \rightarrow \infty} \frac{1}{2} k_{i}\left(\left\|\boldsymbol{l}_{i}\right\|^{2}+l_{0 i}^{2}\right)-k_{i}\left\|\boldsymbol{l}_{i}\right\| l_{0 i}-\left\|\boldsymbol{l}_{i}\right\|\left\|\overline{\boldsymbol{f}}_{i}\right\|= \\
& \lim _{\|\boldsymbol{x}\| \rightarrow \infty}\left\|\boldsymbol{l}_{i}\right\|^{2}\left(\frac{k_{i}}{2}+\frac{k_{i} l_{0 i}^{2}}{2\left\|\boldsymbol{l}_{i}\right\|^{2}}-\frac{k_{i} l_{0 i}}{\left\|\boldsymbol{l}_{i}\right\|}-\frac{\left\|\overline{\boldsymbol{f}}_{i}\right\|}{\left\|\boldsymbol{l}_{i}\right\|}\right)=+\infty .
\end{aligned}
$$

Based on this results and on Theorem 1.15 of [2], we can say that function (3) has a global minimum. Now we can look for this minimum among the stationary points, i.e., where the 
gradient $\nabla V(\boldsymbol{x})=\mathbf{0}$, and among the points where (1) is not differentiable [3].

It is clear that $\nabla \bar{V}(\boldsymbol{x})=\mathbf{0}$ only if $\boldsymbol{v}=\mathbf{0}, \boldsymbol{p}_{R 1}=\overline{\boldsymbol{p}}_{R 1}$ and $t_{L} \boldsymbol{R}_{L} \boldsymbol{e}_{1} \times \overline{\boldsymbol{R}}_{L} \boldsymbol{e}_{1}=\mathbf{0}$. Regarding $V_{i}(\boldsymbol{x})$, let us consider its gradient with respect to the cable configuration $l_{i}$ :

$$
\nabla_{\boldsymbol{l}_{i}} V_{i}(\boldsymbol{x})=\frac{\partial V_{i}(\boldsymbol{x})}{\partial \boldsymbol{l}_{i}}=k_{i}\left(\left\|\boldsymbol{l}_{i}\right\|-l_{0 i}\right) \frac{\boldsymbol{l}_{i}^{\top}}{\left\|\boldsymbol{l}_{i}\right\|}-\overline{\boldsymbol{f}}_{i}^{T}
$$

Then, $\nabla_{\boldsymbol{l}_{i}} V_{i}(\boldsymbol{x})=\mathbf{0}$ if and only if

$$
k_{i}\left(\left\|\boldsymbol{l}_{i}\right\|-l_{0 i}\right) \frac{\boldsymbol{l}_{i}^{\top}}{\left\|\boldsymbol{l}_{i}\right\|}=\boldsymbol{f}_{i}=\overline{\boldsymbol{f}}_{i} .
$$

Condition (6) holds in two different cases:

a) $\frac{k_{i}\left(\left\|\boldsymbol{l}_{i}\right\|-l_{0 i}\right)}{\left\|\boldsymbol{l}_{i}\right\|}=\left\|\overline{\boldsymbol{f}}_{i}\right\|$ and $\boldsymbol{l}_{i}=\frac{\overline{\boldsymbol{f}}_{i}}{\left\|\overline{\boldsymbol{f}}_{i}\right\|}$, for which $\left\|\boldsymbol{l}_{i}\right\|>l_{0 i}$ and $\boldsymbol{l}_{i}^{\top} \overline{\boldsymbol{f}}_{i}>0$;

b) $\frac{k_{i}\left(\left\|\boldsymbol{l}_{i}\right\|-l_{0 i}\right)}{\left\|\boldsymbol{l}_{i}\right\|}=-\left\|\overline{\boldsymbol{f}}_{i}\right\|$ and $\boldsymbol{l}_{i}=-\frac{\overline{\boldsymbol{f}}_{i}}{\left\|\overline{\boldsymbol{f}}_{i}\right\|}$, for which $\left\|\boldsymbol{l}_{i}\right\|<l_{0 i}$ and $\boldsymbol{l}_{i}^{\top} \overline{\boldsymbol{f}}_{i}<0$.

The previous two cases have a straightforward physical interpretation. Since the cables are modeled as a spring, they can produce a force at a certain point both being stretched in the same direction of the force itself, as in case a), or being compressed in the opposite direction, as in case b). However in this work we consider only case a) because case b) is not practicably feasible for cables, thus out of our region of interest.

Therefore $\nabla V(\boldsymbol{x})=\mathbf{0}$ if $\boldsymbol{x} \in \mathscr{X}_{\nabla \mathbf{0}}^{a} \cup \mathscr{X}_{\nabla \mathbf{0}}^{b}$ where

a) $\mathscr{X}_{\nabla \mathbf{0}}^{a}=\left\{\boldsymbol{x} \mid \boldsymbol{v}=\mathbf{0}, \boldsymbol{p}_{R 1}=\overline{\boldsymbol{p}}_{R 1}, t_{L} \boldsymbol{R}_{L} \boldsymbol{e}_{1} \times \overline{\boldsymbol{R}}_{L} \boldsymbol{e}_{1}=\right.$ $\left.\mathbf{0}, \frac{k_{i}\left(\left\|\boldsymbol{l}_{i}\right\|-l_{0 i}\right)}{\left\|\boldsymbol{l}_{i}\right\|}=\left\|\overline{\boldsymbol{f}}_{i}\right\|, \boldsymbol{l}_{i}=\frac{\overline{\boldsymbol{f}}_{i}}{\left\|\overline{\boldsymbol{f}}_{i}\right\|}\right\}$

b) $\mathscr{X}_{\nabla 0}^{b}=\left\{\boldsymbol{x} \mid \boldsymbol{v}=\mathbf{0}, \boldsymbol{p}_{R 1}=\overline{\boldsymbol{p}}_{R 1}, t_{L} \boldsymbol{R}_{L} \boldsymbol{e}_{1} \times \overline{\boldsymbol{R}}_{L} \boldsymbol{e}_{1}=\right.$ $\left.\mathbf{0}, \frac{k_{i}\left(\left\|\boldsymbol{l}_{i}\right\|-l_{0 i}\right)}{\left\|\boldsymbol{l}_{i}\right\|}=-\left\|\overline{\boldsymbol{f}}_{i}\right\|, \boldsymbol{l}_{i}=-\frac{\overline{\boldsymbol{f}}_{i}}{\left\|\overline{\boldsymbol{f}}_{i}\right\|}\right\}$

However, $\boldsymbol{x} \in \mathscr{X}_{\nabla \mathbf{0}}^{b}$ can not be the global minima since we can show that $V\left(\boldsymbol{x}_{a}\right)<V\left(\boldsymbol{x}_{b}\right)$ with $\boldsymbol{x}_{a} \in \mathscr{X}_{\nabla \mathbf{0}}^{a}$ and $\boldsymbol{x}_{b} \in \mathscr{X}_{\nabla \mathbf{0}}^{b}$. This comes from the fact that in (1), $-\boldsymbol{l}_{i}^{\top} \overline{\boldsymbol{f}}_{i}<0$ and $-\boldsymbol{l}_{i}^{\top} \overline{\boldsymbol{f}}_{i}>0$ for $\boldsymbol{x} \in \mathscr{X}_{\nabla \mathbf{0}}^{a}$ and $\boldsymbol{x} \in \mathscr{X}_{\nabla \mathbf{0}}^{b}$, respectively.

Finally we have to check the non-differentiable points of (1), namely the state $\boldsymbol{x} \in \mathscr{X}_{\boldsymbol{l}_{i} 0}=\left\{\boldsymbol{x} \mid\left\|\boldsymbol{l}_{i}\right\|=0\right.$ for $\left.i=1,2\right\}$. Notice that this condition is out of our domain of interest. Nevertheless, also in this case we can show that $V\left(\boldsymbol{x}_{a}\right)<V\left(\boldsymbol{x}_{\boldsymbol{l}_{i} 0}\right)$. Indeed, $\bar{V}\left(\boldsymbol{x}_{a}\right)=\bar{V}\left(\boldsymbol{x}_{l_{i} 0}\right)$ and

$$
\begin{aligned}
V_{i}\left(\boldsymbol{x}_{\boldsymbol{l}_{i} 0}\right) & =\frac{1}{2} k_{i} l_{0 i}^{2} \\
V_{i}\left(\boldsymbol{x}_{a}\right) & =\frac{1}{2} k_{i}\left(\left\|\boldsymbol{l}_{i}\right\|^{2}+l_{0 i}^{2}-2\left\|\boldsymbol{l}_{i}\right\| l_{0 i}\right)-\boldsymbol{l}_{i}^{\top} k_{i}\left(\left\|\boldsymbol{l}_{i}\right\|-l_{0 i}\right) \frac{\boldsymbol{l}_{i}}{\left\|\boldsymbol{l}_{i}\right\|} \\
& =\frac{1}{2} k_{i}\left\|\boldsymbol{l}_{i}\right\|^{2}+\frac{1}{2} k_{i} l_{0 i}^{2}-k_{i}\left\|\boldsymbol{l}_{i}\right\| l_{0 i}-k_{i}\left\|\boldsymbol{l}_{i}^{2}\right\|+k_{i}\left\|\boldsymbol{l}_{i}\right\| l_{0 i} \\
& =\frac{1}{2} k_{i} l_{0 i}^{2}-\frac{1}{2} k_{i}\left\|\boldsymbol{l}_{i}\right\|^{2} .
\end{aligned}
$$

Thus $V_{i}\left(\boldsymbol{x}_{a}\right)<V_{i}\left(\boldsymbol{x}_{l_{i} 0}\right)$.

We can finally conclude that $\boldsymbol{x} \in \mathscr{X}_{\nabla \mathbf{0}}^{a}$ is the global minima of (1). Furthermore, with a similar reasoning to the proof of Theorem 2 of the manuscript, we can show that $\mathscr{X}_{\nabla \mathbf{0}}^{a}=$ $\mathscr{X}\left(0, \overline{\boldsymbol{q}}_{L}\right)$ for $t_{L}=0$ and $\mathscr{X}_{\nabla \mathbf{0}}^{a}=\mathscr{X}^{+}\left(t_{L}, \overline{\boldsymbol{q}}_{L}\right)$ for $t_{L}>0$, proving the first point of Proposition 1.
For the last point, let us define the function $V^{\prime}(\boldsymbol{x})$ as in (1) but without $V_{0}$. Then, in order to obtain $V(\boldsymbol{x})>0$, we can simply set

$$
V_{0}>\min _{\boldsymbol{x}}\left(V^{\prime}(\boldsymbol{x})\right)=V^{\prime}\left(\boldsymbol{x}_{a}\right)
$$

with $\boldsymbol{x}_{a} \in \mathscr{X}_{\nabla \mathbf{0}}^{a}$. 


\section{Load Pose Regulation}

Given the desired load configuration of equilibrium $\bar{p}_{L}=$ $\left[\begin{array}{lll}0.3 & 0.3 & 0.2\end{array}\right]^{\top}[\mathrm{m}], \bar{\psi}=\pi / 7[\mathrm{rad}]$ and $\bar{\theta}=\pi / 8[\mathrm{rad}]$, we performed several simulations with $\pi_{A} \in \Pi_{A}\left(\overline{\boldsymbol{q}}_{L}\right)$ computed for the cases: 1) $\left.\left.t_{L}=1.5>0,2\right) t_{L}=0,3\right) t_{L}=-1<0$,

To study the stability of the equilibrium configuration for the different values of $t_{L}$, we initialized the system in two different initial configurations and we observed the evolution of the system. We observed that for case

1) the system final configuration belongs to $\mathscr{X}^{+}\left(t_{L}, \overline{\boldsymbol{q}}_{L}\right)$. Figure 1 shows the system configuration evolution for the two different initial conditions. The final state of the two trajectories is the same;

2 ) the system final configuration belongs to $\mathscr{X}\left(0, \overline{\boldsymbol{q}}_{L}\right)$, and depends on the system initial state. Figure 2 shows the system configuration evolution in these cases;

3 ) the system does not converge to $\mathscr{X}^{-}\left(t_{L}, \overline{\boldsymbol{q}}_{L}\right)$ even initializing it very close. Figure 3 shows the system configuration evolution in these cases.

The previous plots integrate the ones provided [1] showing the complete evolution of the main quantities of the system for two particular initial conditions.
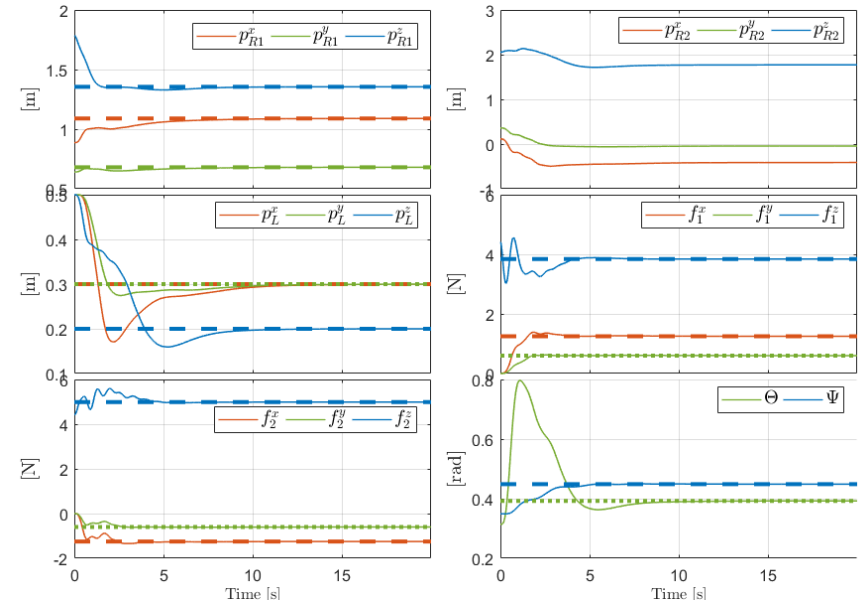

(a) First initial condition.
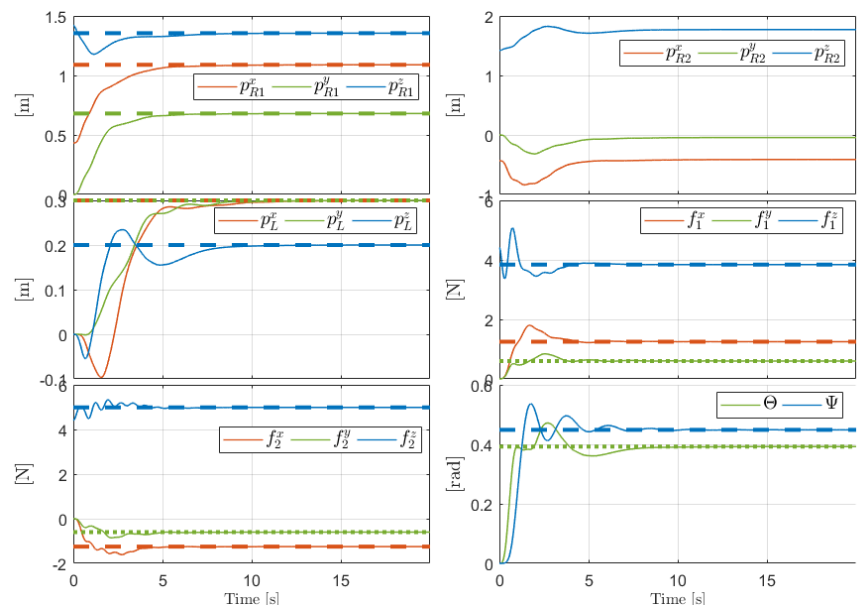

(b) Second initial condition.

Fig. 1: Evolution of the system variables for $t_{L}=1.5[\mathrm{~N}]$ starting from two different initial conditions. The positions of the robots center of masses (which coincide with the cables attaching points) are shown, together with the position of the load center of mass and its yaw and pitch angles. The reference signals are displayed with dotted lines of the same color. 

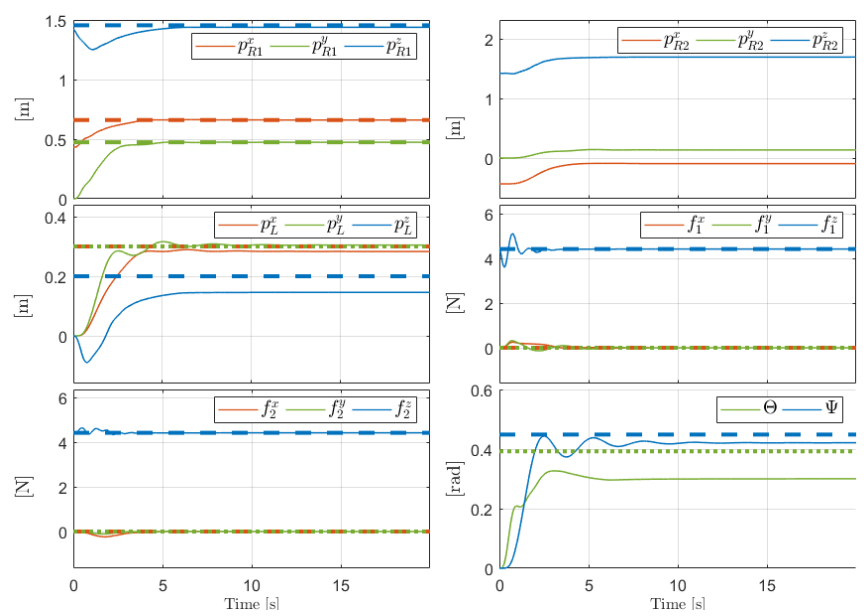

(a) First initial condition.
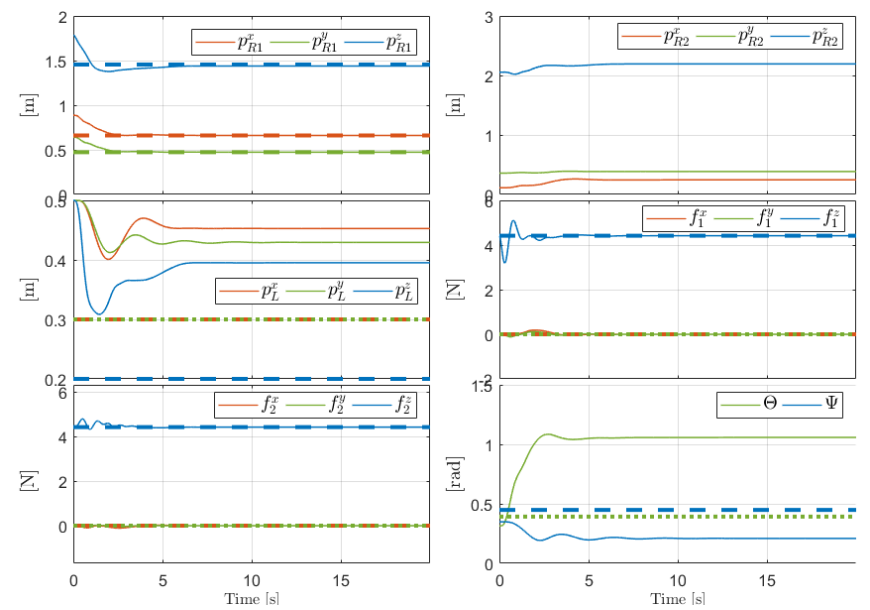

(b) Second initial condition.

Fig. 2: Evolution of the system variables for $t_{L}=0[\mathrm{~N}]$ starting from two different initial conditions.
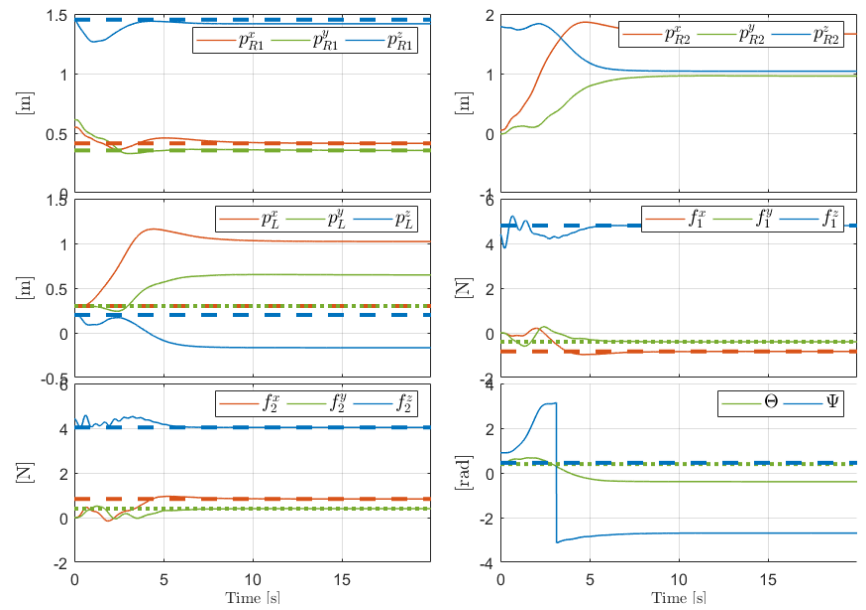

(a) First initial condition.
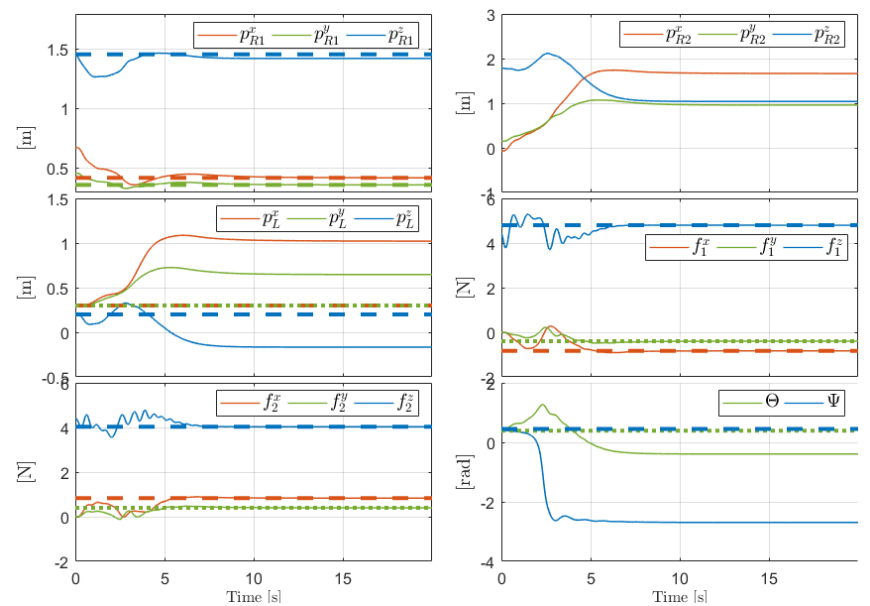

(b) Second initial condition.

Fig. 3: Evolution of the system variables for $t_{L}=-1[\mathrm{~N}]$ starting from two different initial conditions. 


\section{LOAD TRANSPORTATION}

Considering a time-varying control input, we defined $\pi_{A 1}(t)$ such that the master robot follows a 5th-order polynomial trajectory in the three directions (rest to rest with condition of zero acceleration at the initial and final points) starting from an initial position of $\left[\begin{array}{lll}1.18 & 0.72 & 2.2\end{array}\right]^{\top}[\mathrm{m}]$. The trajectory covers $4[\mathrm{~m}]$ along each of the the three directions in $30[\mathrm{~s}]$. The particular $\pi_{A}(t)$, with $\pi_{A 2}(t)=\bar{\pi}_{A 2}^{\prime}$ and $\pi_{A 1}\left(t_{f}\right)=\bar{\pi}_{A 1}^{\prime}$, brings the load in the configuration $\bar{p}_{L}=\left[\begin{array}{llll}4.5 & 4.5 & 5.0\end{array}\right]^{\top}[\mathrm{m}]$, $\bar{\psi}=\pi / 9[\mathrm{rad}]$ and $\bar{\theta}=\pi / 8[\mathrm{rad}]$. In Fig. 5 we show the results of the simulations in ideal conditions. We notice that, once the final input $\pi_{A 1}\left(t_{f}\right)=\bar{\pi}_{A 1}^{\prime}$ with $t_{L}>0[\mathrm{~N}]$ is set, the system successfully transports the load between the two points stopping at the desired configuration, as shown in Fig. 4(a). For $t_{L}=0[\mathrm{~N}]$ instead, the final load attitude depends on the particular motion, and it is in general different from the desired one, as shown in Fig. 4(b). Finally, as one can see in Fig. 4(c), when $t_{L}<0[\mathrm{~N}]$ the final configuration of the system does not correspond to the desired one, since it was an unstable equilibrium. Notice in Fig. 4(a) how the error on the load trajectory remains sufficiently small for all the transportation, and goes to zero at the end of the task. In Fig. 5(a), 5(b) and 5(c) we show the results for a similar task, for $t_{L}>0$, $t_{L}=0$ and $t_{L}<0$, respectively. In this case the trajectory is followed at a higher speed, since it is completed in $4[\mathrm{~s}]$. Consequently, as one can see in Fig. 5(a), the system moves faster and the tracking error increases. However it remains always bounded and the stability during the transportation is preserved. Furthermore, one could tune the admittance controller parameters of the slave robot to achieve better results if needed.
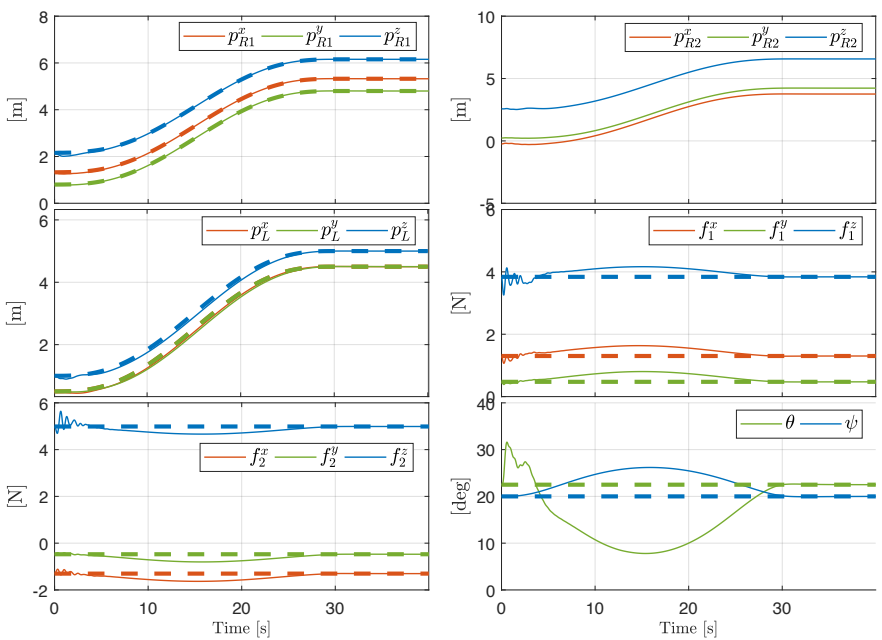

(a) $t_{L}=1.5[\mathrm{~N}]$.
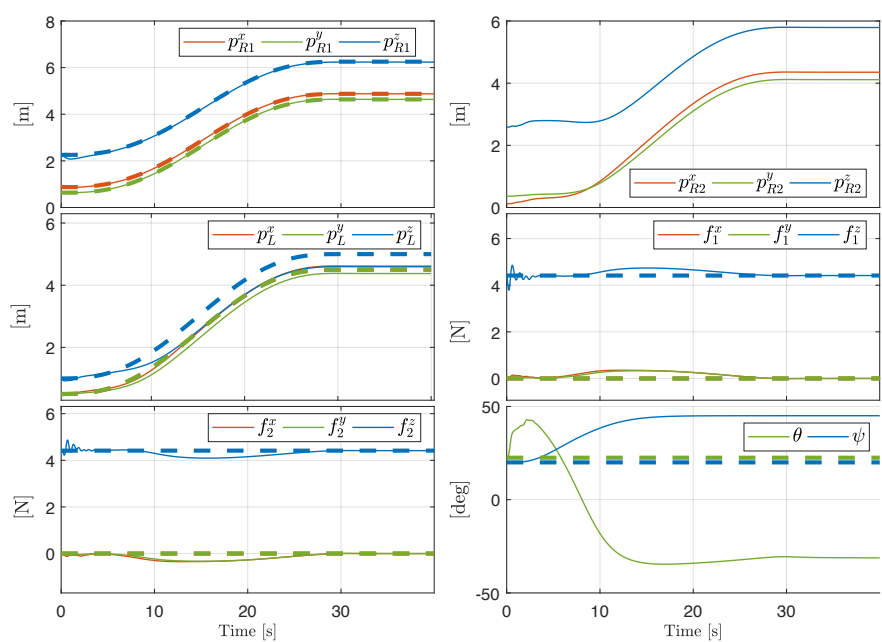

(b) $t_{L}=0[\mathrm{~N}]$.
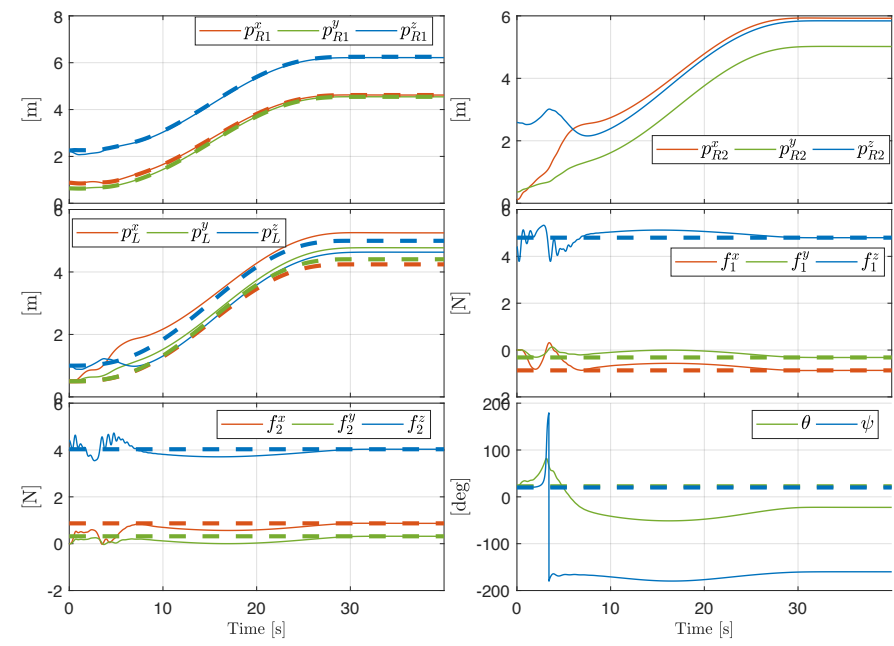

(c) $t_{L}=-1[\mathrm{~N}]$.

Fig. 4: Evolution of the system variables during transportation for the three different values of internal force. 

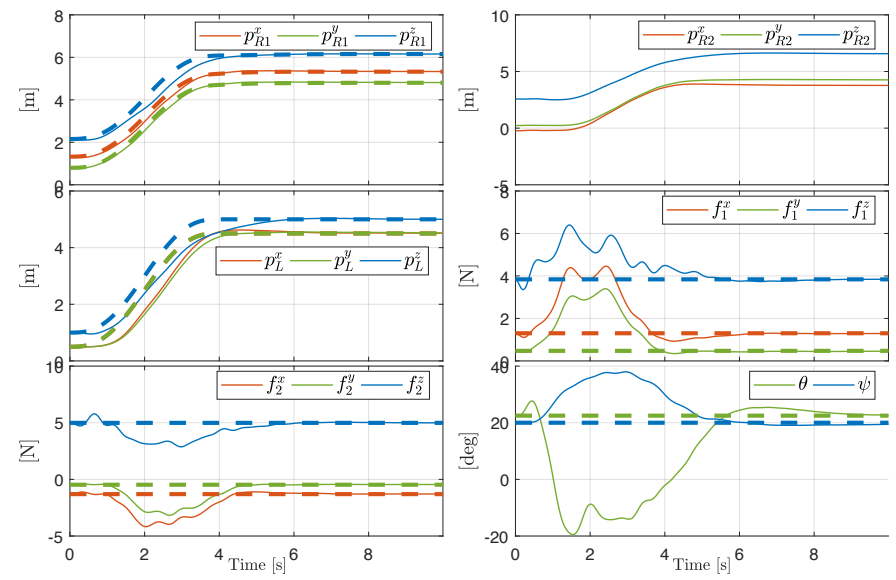

(a) $t_{L}=1.5[\mathrm{~N}]$.
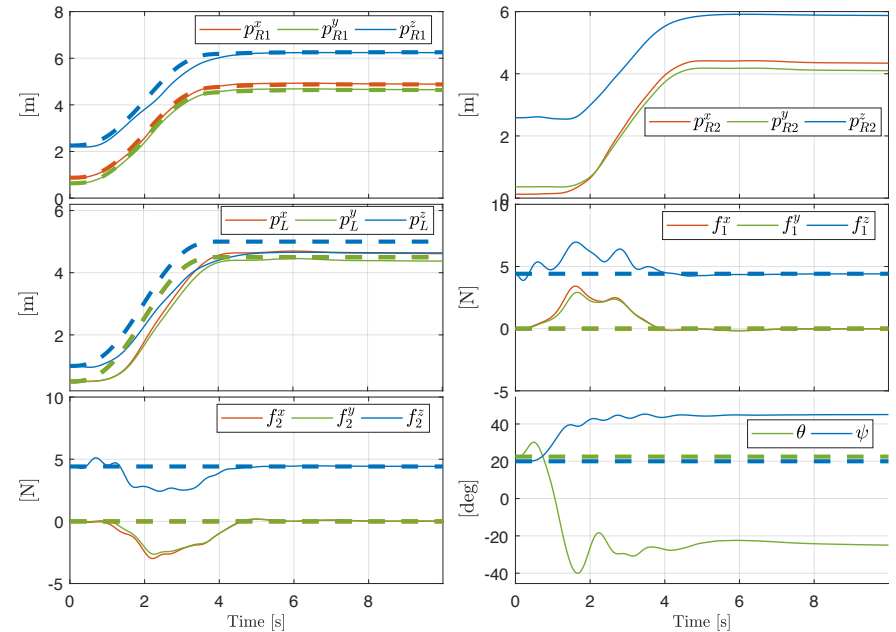

(b) $t_{L}=0[\mathrm{~N}]$.
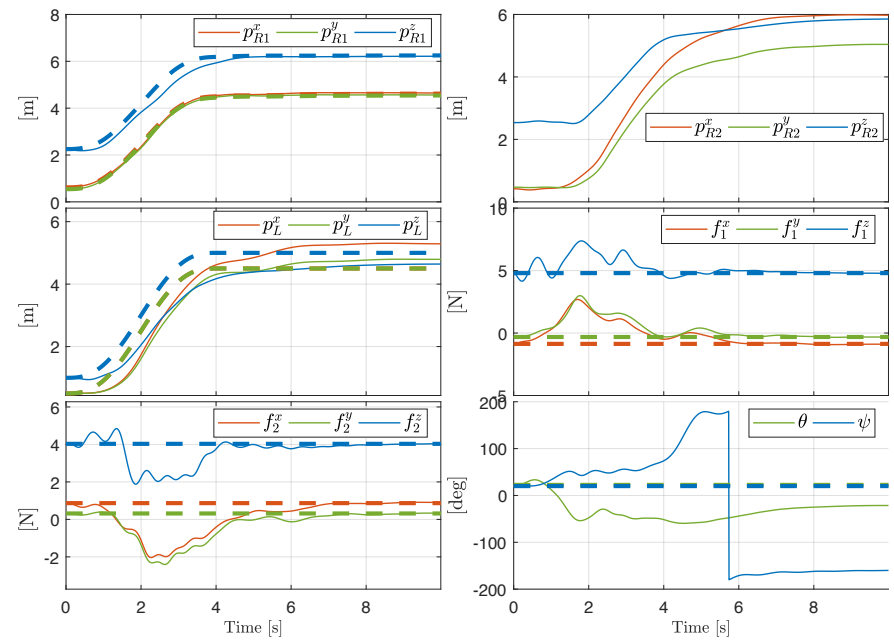

(c) $t_{L}=-1[\mathrm{~N}]$.

Fig. 5: Evolution of the system variables during transportation for the three different values of internal force. 

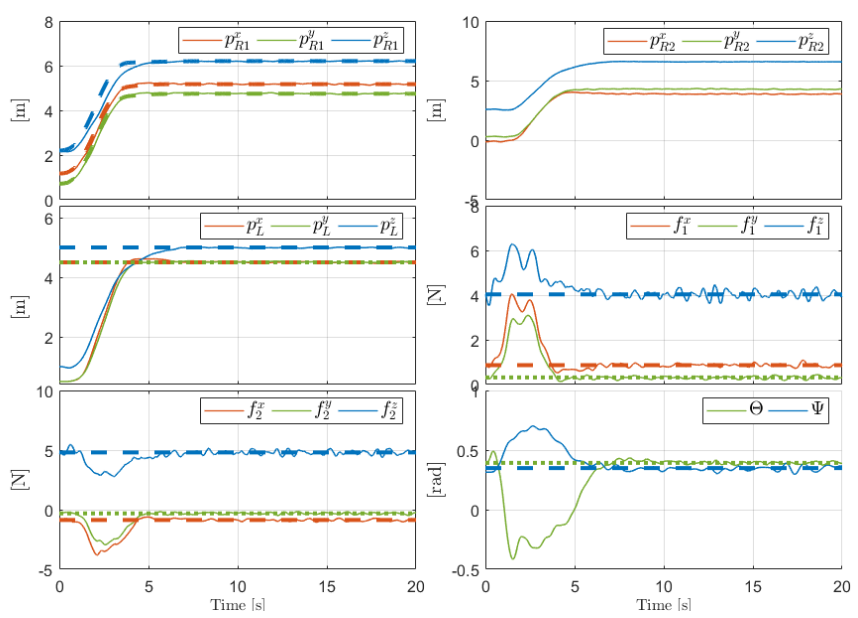

Fig. 6: Simulation result with noisy measurements.

\section{NON-IDEAL CONDITIONS}

In the following, we test the robustness of the proposed method against noise in the measured state and model parameter uncertainties. The following simulations consider the transportation scenario presented in Sec. V, where the trajectory is performed in $4[\mathrm{~s}]$.

\section{A. Noisy Measurements}

In Fig. 6 we report the results of a simulation where Gaussian noise is added to the estimated state of the robots and to the measured cable force, in order to simulate real sensors. In particular, the noise variances on the aerial vehicle position, velocity and measured cable force are equal to $0.005[\mathrm{~m}]$, $0.01[\mathrm{~m} / \mathrm{s}]$ and $0.01[\mathrm{~N}]$, respectively. From the plots one can see that, even in the presence of noise, the system is able to bring the load to the desired pose showing only very small oscillations.

\section{B. Noisy Measurements and Parametric Uncertainties}

In Fig. 7 both measurement noise and parametric uncertainties are considered. In particular, the rest length of the cables, the cables anchoring points positions with respect to the center of mass of the load (or equivalently the position of the center of mass of the load) and the mass of the load are uncertain parameters. In other words, we put ourselves in a condition in which the real parameters and the nominal ones do not perfectly match. In particular, the known cables rest length has been set $5 \%$ greater than the real one, the load mass used to generate the constant control input $\pi_{A}$ is $20 \%$ greater than the real one, and the anchoring points positions in body frame have been chosen as follows: ${ }^{L} \boldsymbol{b}_{1}=$ $\left[\begin{array}{lll}0.5 & 0.01 & 0.02\end{array}\right]^{\top}[\mathrm{m}],{ }^{{ }^{L} \boldsymbol{b}_{2}}=\left[\begin{array}{lll}-0.47 & 0.02 & 0.03\end{array}\right]^{\top}[\mathrm{m}]$. With this simulation we want to show that the proposed algorithm is robust to uncertainties on the parameters in the sense that the system final equilibrium will be clearly different from the desired one, but the robots are still perfectly capable of performing the object transportation task in a stable way, as guaranteed by the system passivity. Fig. 7 shows the results of the simulation during the transportation. As on can see, the
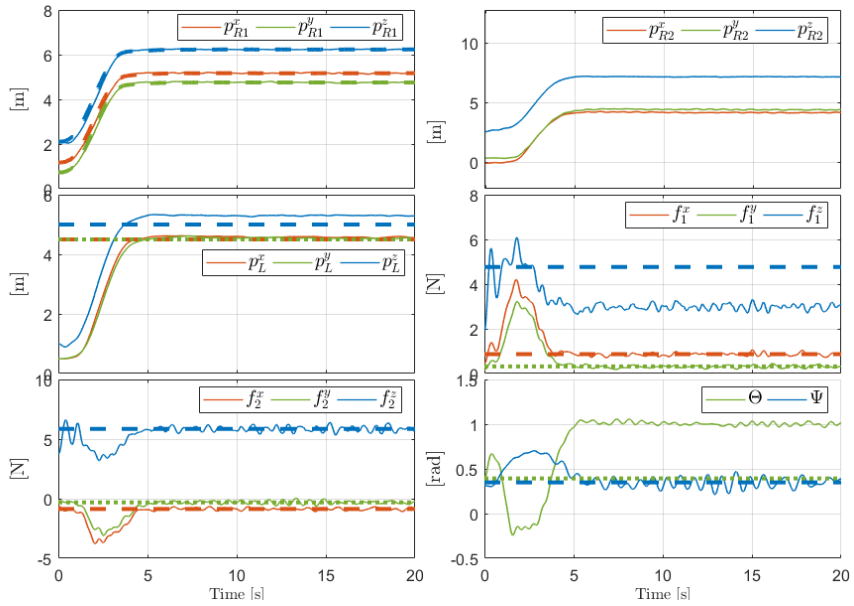

Fig. 7: Evolution of the main system variables for the transportation can in the presence of noise and model uncertainties.

passive nature of the closed loop system makes the system state and output completely stable and converging to a constant equilibrium, that is of course different from the desired one because of the wrong parameter used. An adaptive approach could be used to reduce the effect of this

\section{Sensitivity to Load Mass Uncertainty}

We performed several simulations varying the mass of the load known by the controller with respect to the real mass. Figure 8 displays how the load position and attitude errors at steady state, $e_{L}^{p}$ and $e_{L}^{a}$, change when the real mass is not exactly known. In particular, the errors are defined as:

$$
\begin{aligned}
& e_{L}^{p}=\left\|p_{L}-\bar{p}_{L}\right\| \\
& e_{L}^{a}=\|\theta-\bar{\theta}\|+\|\psi-\bar{\psi}\| .
\end{aligned}
$$

The load starts from the configuration given by: $p_{L}(0)=$ $\left[\begin{array}{lll}0.5 & 0.5 & 1\end{array}\right]^{\top}[\mathrm{m}], \psi=\pi / 10[\mathrm{rad}], \theta=\pi / 8[\mathrm{rad}]$ and $t_{L}=$ $1.5[\mathrm{~N}]$. The desired final configuration is given by $\bar{p}_{L}=$ $\left[\begin{array}{lll}0.5 & 0.5 & 1\end{array}\right]^{\top}[\mathrm{m}], \bar{\theta}=\pi / 9[\mathrm{rad}], \bar{\psi}=\pi / 8[\mathrm{rad}], t_{L}=1.5[\mathrm{~N}]$. Calling $m_{L}^{\prime}$ the known mass, we compute it as $m_{L}^{\prime}=\Delta m \cdot m_{L}$ where $\Delta m$ is the relative mass increment. Figure 8 shows $e_{L}^{p}$ and $e_{L}^{a}$ with respect to $\Delta m$. The larger the parametric uncertainty on the load mass, the more the errors increase, too. However one can notice that even with an uncertainty grater than the $25 \%$ the system still remains stable. After the value $\Delta m=1.3$ the system becomes unstable. Nevertheless, we remark that the mass of the load is one of the parameters that can be known with very good precision, also using an online estimation algorithm.

\section{Sensitivity to Anchoring Point Position Uncertainty}

As an additional study of the robustness of the proposed method, in Fig. 9 we show the load position and attitude errors at steady state when the parametric uncertainty is on 

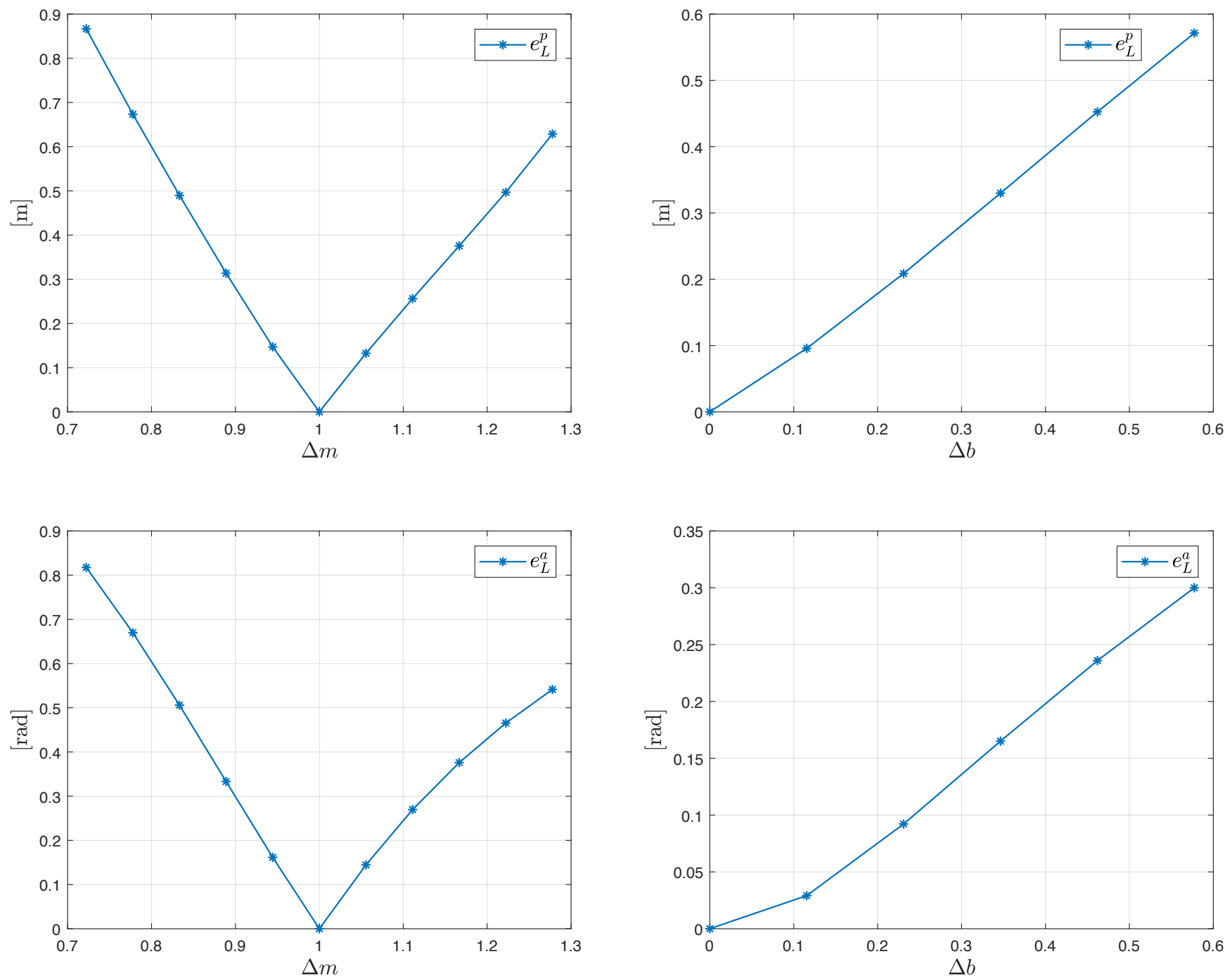

Fig. 8: Load position and attitude errors when the load mass known by the controller differs from the real one.

Fig. 9: Load position and attitude errors when the anchoring points position on the load known by the controller differs from the real one.

the position of the cables anchoring points on the load. In particular, the known anchoring positions are given by

$$
\begin{aligned}
& { }^{L} \boldsymbol{b}_{1}^{\prime}={ }^{L} \boldsymbol{b}_{1}+\left[\begin{array}{l}
1 \\
1 \\
1
\end{array}\right] \Delta b\left\|^{L} \boldsymbol{b}_{1}\right\| \\
& { }^{L} \boldsymbol{b}_{2}^{\prime}={ }^{L} \boldsymbol{b}_{2}+\left[\begin{array}{c}
-1 \\
1 \\
1
\end{array}\right] \Delta b\left\|^{L} \boldsymbol{b}_{1}\right\|,
\end{aligned}
$$

where $\Delta b \in \mathbb{R}_{>0}$. The system starts from the configuration given by $p_{L}(0)=\left[\begin{array}{lll}0.5 & 0.5 & 1\end{array}\right]^{\top}[\mathrm{m}], \psi=\pi / 10[\mathrm{rad}], \quad \theta=$ $\pi / 8[\mathrm{rad}]$ and $t_{L}=1.5[\mathrm{~N}]$. The desired final configuration is given by $\bar{p}_{L}=\left[\begin{array}{lll}0.5 & 0.5 & 1\end{array}\right]^{\top}[\mathrm{m}], \bar{\theta}=\pi / 9[\mathrm{rad}], \bar{\psi}=\pi / 8[\mathrm{rad}]$, $t_{L}=1.5[\mathrm{~N}]$. Also in this case, as expected, the larger the parametric uncertainty on the considered quantities, the more the errors increase, too. However, with the considerable variation of $\Delta b=0.5$ the system still remains stable. 


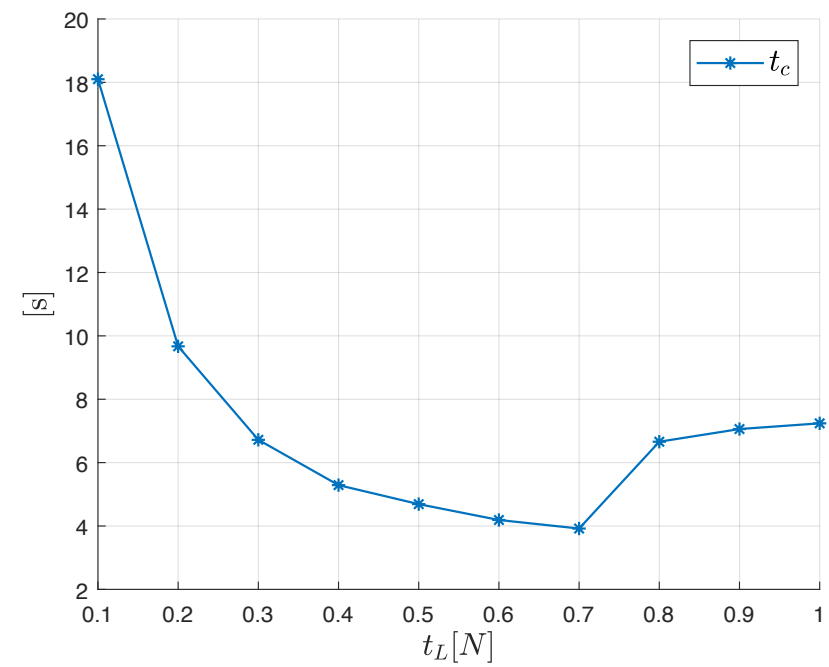

Fig. 10: Convergence time of the load position and attitude errors when the $t_{L}$ increases.

\section{EFFEcts of the InTERnAl Force AlONG THE LOAD}

In the manuscript we saw that to make a desired load configuration asymptotically stable, one has to compute the proper control input $\boldsymbol{\pi}_{A}\left(\overline{\boldsymbol{q}}, t_{L}\right)$ choosing $t_{L}>0$. In the following, we shall analyze the effects of the intensity of the internal force on the system behavior. In this way we can better decide the value of $t_{L}$. In particular, in the following, we shall analyze the relations between $t_{L}$ and convergence time, and between $t_{L}$ and required total thrust in the equilibrium configuration.

\section{A. Internal Force and Convergence Time}

If the internal force $t_{L}=0[\mathrm{~N}]$ the load does not in general converge to its desired pose, which instead happens for $t_{L}>0$. However, it is interesting to see how the convergence rate behaves changing the intensity of the internal force. In Fig. 10, we show how the convergence time of the load position and attitude, defined by $t_{c}$, varies when increasing the internal force. Here $t_{c}=\min \left\{t_{c}^{a}, t_{c}^{p}\right\}$, where $t_{c}^{a}$ is the time after which $e_{L}^{a}$ remains below $5\left[^{0}\right]$, while $t_{c}^{p}$ is the time after which $e_{L}^{p}$ remains below $0.02[\mathrm{~m}]$. The initial and the final desired load configurations are the same as before. Notice that for $t_{L}=0$ the convergence time is in general infinite. One can notice that increasing $t_{L}$ up to $0.7[\mathrm{~N}], t_{c}$ decreases. However, after this value, $t_{c}$ starts to increase due to the appearance of some larger oscillations that takes more time to be damped. In any case, this study shows that even a minimal internal force of $0.1[\mathrm{~N}]$ is enough to obtain asymptotically stability for which an almost negligible increase of total thrust is required.

\section{B. Internal Force and Total Thrust}

Since the internal force, necessary to make the load converge to the desired pose, implies an additional energy consumption for the robots, we evaluated the amount of additional thrust required when the internal force increases. Given a

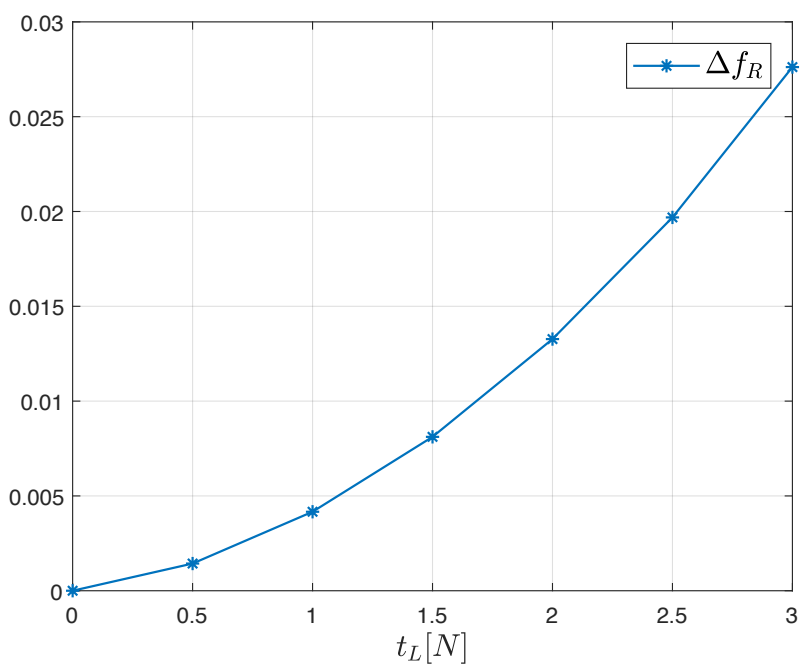

Fig. 11: Additional thrust required by the two robots to stabilize the load when $t_{L}$ increases.

certain desired load pose, Fig. 11 shows the relative increase of total thrust, $\Delta f_{R}$, augmenting the intensity of the internal force with respect to the total thrust required by the case with zero internal force. In particular $\Delta f_{R}$ is computed as

$$
\Delta f_{R}\left(t_{L}\right)=\frac{f_{R}\left(t_{L}\right)-f_{R}^{0}}{f_{R}^{0}}
$$

where $f_{R}\left(t_{L}\right)$ is the the sum of the thrusts required by the two vehicles at steady state to stabilize the load at a certain load configuration with a certain value of $t_{L}$, and $f_{R}^{0}=f_{R}(0)$.

One can notice that even imposing $t_{L}=1[\mathrm{~N}]$, much higher than the real internal force required to stabilize the system, the $\Delta f_{R}$ is below the 0.005 , i.e., the total extra thrust is lower than the $0.5 \%$ of the total thrust required with $t_{L}=0[\mathrm{~N}]$.

In any case we remark that the proposed control method is still applicable for $t_{L}=0[\mathrm{~N}]$. The system is proven to be still stable, but will not clearly asymptotically converge to the desired pose.

\section{REFERENCES}

[1] M. Tognon, C. Gabellieri, L. Pallottino, and A. Franchi, "Aerial comanipulation with cables: The role of internal force for equilibria, stability, and passivity," IEEE Robotics and Automation Letters, Special Issue on Aerial Manipulation, 2018.

[2] R. Horst, P. M. Pardalos, and N. V. Thoai, Introduction to global optimization. Springer Science \& Business Media, 2000.

[3] K. Eriksson, D. Estep, and C. Johnson, Applied Mathematics Body and Soul: Vol III. Springer-Verlag Publishing, 2003. 